\title{
Evaluations of NAM Forecasts on Midtropospheric Perturbation-Induced Convective Storms over the U.S. Northern Plains
}

\author{
SHIH-YU WANG* AND TSING-CHANG CHEN \\ Department of Geological and Atmospheric Sciences, Iowa State University, Ames, Iowa \\ S. ELWYNN TAYLOR \\ Department of Agronomy, Iowa State University, Ames, Iowa
}

(Manuscript received 14 July 2008, in final form 9 April 2009)

\begin{abstract}
In the U.S. northern plains, summer progressive convective storms that occur in weakly forced environments are often coupled with short-wave perturbations that are embedded in the midlevel northwesterly flow. These midtropospheric perturbations (MPs) are capable of inducing propagating convection that contributes to a majority of the rainfall over the northern plains during July and August. There is a possibility that the difficulties of numerical weather prediction models in forecasting summer convective rainfall over the northern plains are partly attributed to their deficiency in forecasting MPs. The present study tests this possibility through examining operational forecasts by the North American Mesoscale (NAM) model during the summers of 2005 and 2006.

Forecasted MPs exhibit slower propagation speeds and weaker relative vorticity than the observations leading to systematic position errors. Underpredicted vorticity magnitudes weaken horizontal vorticity advection that influences the vorticity tendency throughout the MP life cycle and, in turn, slows the propagation speed of MPs. Moreover, biases of weak ambient flow speed and vortex stretching contribute to the magnitude and propagation speed errors of MPs. Skill scores of precipitation forecasts associated with MPs are low, but can be considerably improved after removing the MP position error that displaces the rainfall pattern. The NAM also tends to underpredict precipitation amounts. A modified water vapor budget analysis reveals that the NAM insufficiently generates atmospheric humidity over the central United States. The shortage of moisture in the forecast reduces the water vapor flux convergence that is part of the precipitation process. The precipitation bias may feed back to affect the MP growth through the bias in heating, thus further slowing the perturbation.
\end{abstract}

\section{Introduction}

Progressive mesoscale convective systems (MCSs) that travel a long distance across the U.S. northern plains are common in midsummer (Johns and Hirt 1987; Bentley and Mote 1998). Such MCSs usually propagate southeastward underneath midlevel northwesterly flow (Johns 1982; Johns and Hirt 1987), a behavior different

\footnotetext{
* Current affiliation: Utah Climate Center, Utah State University, Logan, Utah.

Corresponding author address: Shih-Yu (Simon) Wang, Utah Climate Center, Utah State University, 4820 Old Main Hill, Logan, UT 84322.

E-mail: simon.wang@usu.edu
}

from the common northeastward-moving MCSs in late spring (Johns 1993). Repeated passages of progressive MCSs over the same area, an important factor leading to Midwestern flash floods, have been observed to couple with serial short-wave perturbations in the midtroposphere (e.g., Maddox et al. 1979; Johns 1984; Changnon and Kunkel 1999). Sub-synoptic-scale cyclonic perturbations at midlevels can sustain long-lived, progressive MCSs across the Upper Midwest (e.g., Bosart and Sanders 1981). Trier et al. (2006) noted that a series of preexisting perturbations embedded in the midlevel northwesterly flow induced a series of progressive MCSs in July 2003. These previous findings highlight the difference of such sub-synoptic-scale perturbations from mesoscale convective vortices (MCVs) spawned by MCSs (e.g., Menard and Fritsch 1989). 
Examining the characteristics of similar midtropospheric perturbations (MPs) during the warm seasons of 1997-2006, Wang et al. (2009, hereafter WCC) reported that MPs are recurrent circulation features related to about $60 \%$ of the rainfall in midsummer (July-August) over the Upper Midwest. MPs exhibit a regular life cycle from their initiation over the Rocky Mountains to their demise or merger with a long-wave trough near the East Coast. Upward motion forming ahead of MPs helps induce convection, while their downstream (east-southeastward) propagation creates the progressive property of the associated convective storms. WCC proposed that MPs likely cause northwest flow severe weather outbreaks, which occur under a large-scale ridge with prevailing midlevel northwesterlies (Johns 1982), a type of synoptic circulation described as the "warm season pattern" (Johns 1993). In other words, MPs may play a role in sub-synoptic-scale forcing for progressive MCSs under an environment lacking large-scale forcing.

Large-scale forcing is known as a crucial factor in MCS forecasting. Strong baroclinicity of the environmental flow, often in the forms of apparent vorticity advection and surface frontogenesis, assists in a better prediction of MCS rainfall (Jankov and Gallus 2004). These conditions are common in the "dynamic pattern," which features a migrating synoptic wave west of the storm area (e.g., Uccellini and Johnson 1979; Johns 1993; Hilgendorf and Johnson 1998). However, the warm season pattern in which progressive MCSs occur features weak or no baroclinic forcing at middle and upper levels as supplied by the dynamic pattern (Johns 1993; Stensrud and Fritsch 1994; Coniglio et al. 2004). Low-level synoptic conditions become important to progressive MCSs under the warm season pattern, such as warm advection and thermal instability along a quasistationary front (e.g., Maddox 1983; Johns and Hirt 1987) accompanied by northwesterly wind shear (Coniglio et al. 2004). Forecasts of MCS rainfall in weakly forced environments are usually poor (Olson et al. 1995; Jankov and Gallus 2004; Liu et al. 2006). Because MPs provide midlevel forcing under the weak synoptic environment, and because MPs are related to a large population of progressive MCSs (WCC), such poor forecasts imply that MPs are not handled properly in current forecast models.

Accurate forecasts of MCS rainfall require good predictions of the occurrence, timing, and position of the systems (Jankov and Gallus 2004). All of these elements are particularly crucial in simulating summer precipitation over the central United States due to its distinct propagation and diurnal properties in association with long-lived convective episodes (Carbone et al. 2002; Davis et al. 2003; Tuttle and Davis 2006). However, progres- sive precipitation patterns are quite difficult to simulate. Operational forecast models with coarse grid spacings have been found incapable of replicating the coherent propagating rainfall pattern observed in the Midwest (Davis et al. 2003; Clark et al. 2007). Manipulating cumulus parameterization schemes (CPSs) and reducing grid spacings have been the common approaches for improving summer quantitative precipitation forecasts (QPF) [see Stensrud (2007) for a review]. While problems with CPSs have been found to hurt simulations of propagating precipitation (Davis et al. 2003; Liu et al. 2006), finer grid spacings without employing CPSs were found to work better (e.g., Clark et al. 2007). QPFs are also influenced by how accurately the rain-producing weather systems are predicted. For example, the intensities and positions of simulated midlatitude winter cyclones (Grumm and Siebers 1990; Du et al. 1997), tropical cyclones (Peng and Chang 1996; Marchok et al. 2007), or African easterly waves (Céron and Guérémy 1999) are essential to accurate QPFs over the specific regions. Examining numerical forecasts of MPs is important so that additional contributions to QPF errors for progressive MCSs can be identified.

The purpose of this study is to evaluate the forecasts of MPs and their associated rainfall in the National Centers for Environmental Prediction's (NCEP's) operational North American Mesoscale (NAM) model. Evaluation of operational data is necessary because these routine model outputs affect the largest population of a variety of users. WCC showed that MPs are most frequent and particularly important to rainfall during July and August; thus, the evaluation focuses on these 2 months. A diagnostic approach is adopted in this study. Given the dimension of MPs ( $\sim 1400 \mathrm{~km}$ in wavelength), this study focuses on the subsynoptic characteristics rather than the mesoscale details. The data and the MP selection procedure are introduced in section 2. A couple of MP cases, together with their forecasts by the NAM, are displayed in section 3. Systematic errors of MP forecasts are diagnosed and presented in section 4, and the diagnostic schemes for revealing model errors are also introduced in this section. A summary and concluding remarks are given in section 5 .

\section{Data and methodology}

\section{a. Data sources}

This study uses the North American Regional Reanalysis (NARR) for observational atmospheric data. The NARR was developed by NCEP using the latest Data Assimilation System of the NCEP Eta Model (Black 1994) at 32-km, 45-layer resolutions with 3-hourly 
output. The quality of the NARR has been demonstrated to be very close to the observations made from rain gauges, rawinsonde reports, and surface stations over the continental United States (Mesinger et al. 2006). The NARR data are obtained from the National Climatic Data Center (information online at http:// www.ncdc.

noaa.gov/oa/ncdc.html). The NAM, formerly known as the Eta Model, has 12-km grid spacing with 45 vertical layers and is run 4 times daily with 3-hourly output. In June 2006, the Nonhydrostatic Mesoscale Model of the Weather Research and Forecasting model (WRFNMM; Bernardet et al. 2005) replaced the Eta Model to become the official NAM. Therefore, the 2005 and 2006 periods of this study involve two forecast models, which are designated hereafter as NAM05 and NAM06, respectively. To be comparable to the NARR, the grid spacing of NAM is reduced to $32 \mathrm{~km}$ using bilinear interpolation. The NAM outputs are provided by the National Climatic Data Center (information online at http://nomads.ncdc.noaa.gov/).

The NCEP national precipitation analysis (stage IV) dataset is used as the observed rainfall. The stage IV data are estimated from about 140 Weather Surveillance Radars-1988 Doppler (WSR-88Ds) and more than 3000 rain gauge reports over the continental United States at 4-km spatial resolution (Lin and Mitchell 2005). The spatial resolution of stage IV data is also reduced to $32 \mathrm{~km}$ to be comparable with the NARR and the NAM using procedures employed at NCEP that conserve the total amount of liquid in the domain (Clark et al. 2007). To be consistent with the NAM's 3-hourly accumulated rainfall, the 3-h accumulation of the stage IV precipitation is used herein. The stage IV data are provided by NCEP (information online at http://www.emc.ncep.noaa.gov).

\section{b. MP selection criteria}

Wind fields and the relative vorticity $(\zeta)$ at 600 and $200 \mathrm{hPa}$, analyzed every $3 \mathrm{~h}$, are used to detect MPs through the following procedure developed in WCC:

1) The large-scale circulation at $600 \mathrm{hPa}$ exhibits an anticyclone-ridge over the central-western United States and a long-wave trough over the eastern seaboard. The average wind direction within the detection domain $\left(35^{\circ}-47^{\circ} \mathrm{N}\right.$ and $100^{\circ}-85^{\circ} \mathrm{W}$; Fig. 1a) must be between westerly and northerly $\left(270^{\circ}-360^{\circ}\right)$ at both 600 and $200 \mathrm{hPa}$.

2) To remove gravity waves embedded in the fine-grid spacing (Barnes et al. 1996), a nine-point smoothing is applied to $\zeta$. In the detection domain, a cyclonic perturbation should appear at $600 \mathrm{hPa}$ with the maximum $\zeta$ exceeding $10^{-5} \mathrm{~s}^{-1}$ (after smoothing).
There must be at least five grid points with $\zeta \geq$ $10^{-5} \mathrm{~s}^{-1}$ near the maximum $\zeta$ region, while the grid point containing the maximum $\zeta$ marks the center position of the perturbation. Perturbations satisfying these conditions must last for at least $24 \mathrm{~h}$.

3) The initial point of the perturbation is determined by tracing it back until none of the grid points contain $\zeta \geq 10^{-5} \mathrm{~s}^{-1}$. The initial point must be located west of the detection domain $\left(100^{\circ} \mathrm{W}\right)$. The ending point is decided using the same method as the initial point, or when the perturbation merges with the large-scale trough near the eastern seaboard. The tracking is done manually in order to ensure temporal continuity and follow the detected perturbation outside the detection domain.

4) Through visual inspection of the $200-\mathrm{hPa}$ synoptic pattern, no synoptic-scale troughs $(\geq 2000 \mathrm{~km})$ appear within $10^{\circ}$ radius of the detected perturbation. This procedure is used to reconfirm criterion 1 - that the influence of large-scale synoptic forcing on the selected cases is minimal.

5) To exclude tropopause folds that can inject high potential vorticity air into the midtroposphere, we follow Davis et al. (2002) in an attempt to avoid signatures of shear lines and upper-level fronts by visually filtering horizontally elongated vorticity features at $200 \mathrm{hPa}$.

The same procedure is applied to MP tracking in the NAM forecasts.

The geographic frequency of observed MPs during July and August of 2005-06 is displayed in Fig. 1a. In this particular season, MPs form a high-frequency belt extending from the northwest Rocky Mountains to the Ohio Valley parallel to the midlevel northwesterly flow. The durations and genesis dates of MPs are shown in Fig. 1b. The average duration of MPs is about $48 \mathrm{~h}$, distinctly longer than the common life cycle of MCVs between 3 and $12 \mathrm{~h}$ (Davis et al. 2002). There are $15 \mathrm{MP}$ cases in 2005 and 20 in 2006. Table 1 summarizes the basic characteristics of these MPs, including the date when the MP is mostly within the detection domain, maximum $\zeta$ and diameter, and the zonal distance of maximum precipitation boundary within the $1 \mathrm{~mm} \mathrm{~h}^{-1}$ contour.

\section{Individual cases and the forecasts}

An example event involving three consecutive MPs associated with three progressive MCSs that occurred on 20-22 July 2005 is examined. The synoptic conditions at 0900 UTC 21 July are shown in Fig. 2a in terms of 300-, $600-$, and $900-\mathrm{hPa}$ streamlines superimposed with the stage IV rainfall. A series of short-wave troughs depicting 
(a) $\mathrm{V}(600 \mathrm{hPa}), \mathrm{N}_{\mathrm{F}}(\mathrm{MP})$

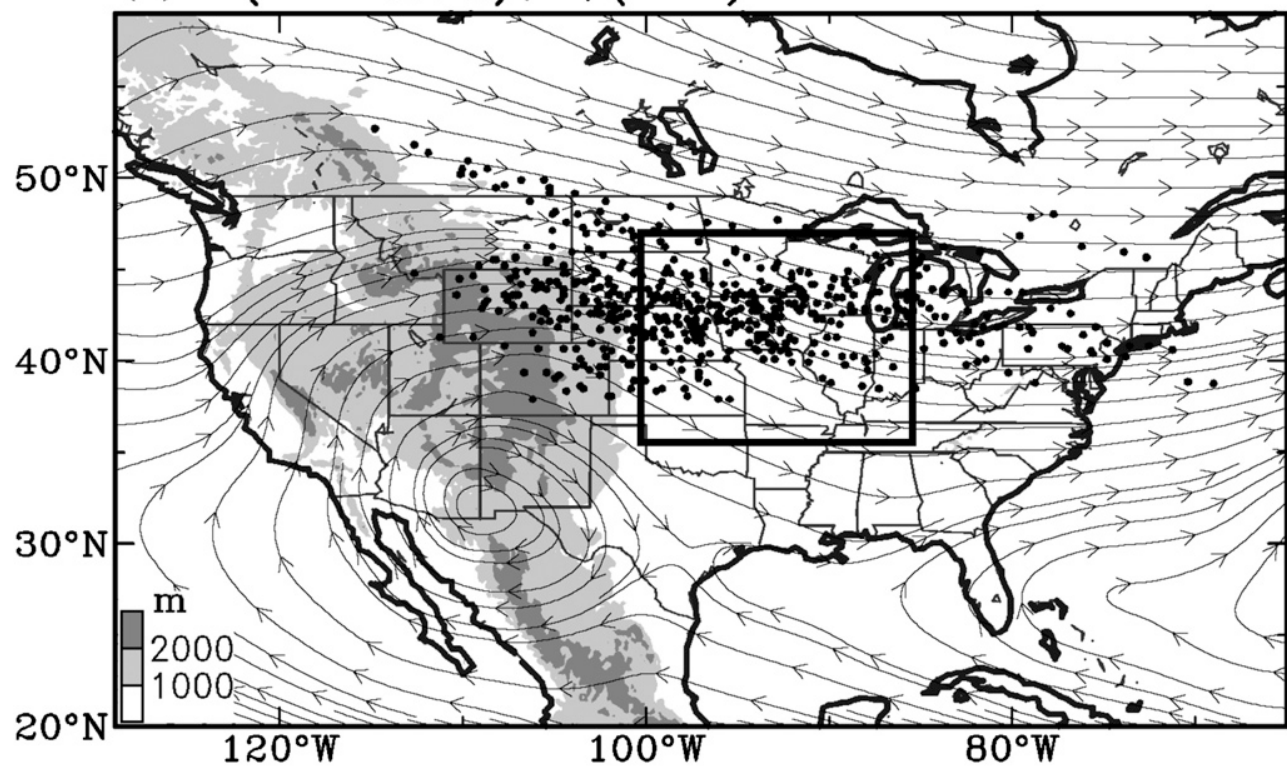

FIG. 1. (a) Mean 600-hPa streamlines (V) during July-August of 2005-06 superimposed on 3-h locations of midtropospheric perturbations $\left(N_{F}\right.$; dots), and (b) the occurrence frequencies of MPs showing their genesis (thin sticks) and duration (thick bars) during the analysis period. Terrain is shaded in (a). The MP detection domain is outlined in (a).

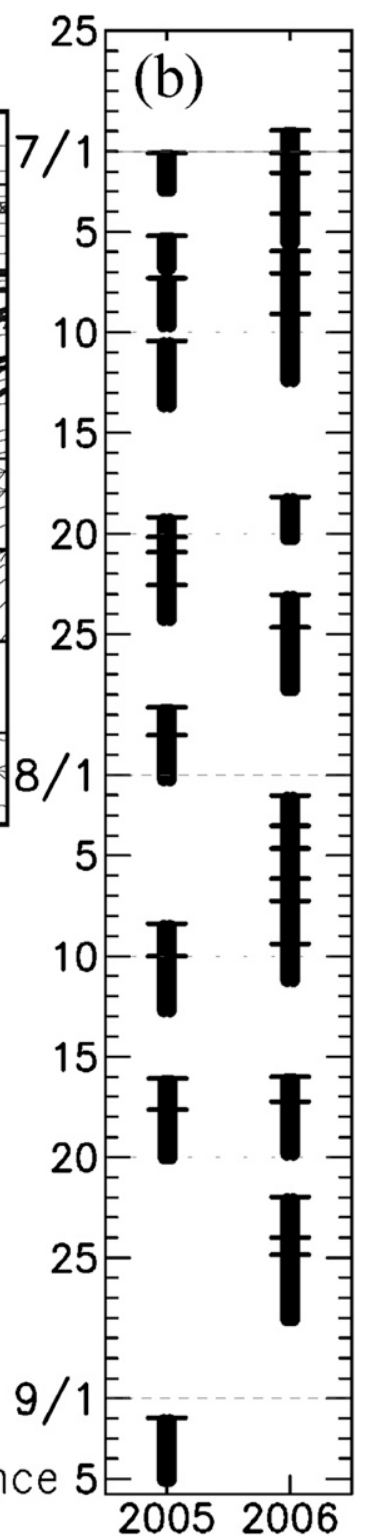

the MPs appear at $600 \mathrm{hPa}$ (marked with the corresponding case number). The MCSs accompanying the first and second MPs (MPs 1 and 2) were propagating southeastward along the midlevel northwesterly flow, while the third storm was close to its initiation time near MP 3 over Montana. The 300-hPa flow shows a wellorganized anticyclone featuring smooth, anticyclonic curvatures where the perturbations/storms are situated. At $900 \mathrm{hPa}$, the flows outline a convergence boundary across these convective storms along the northern terminus of the low-level jet. Except for the MP series at $600 \mathrm{hPa}$, all these synoptic conditions resemble those prone to progressive derechos under the warm season pattern (Johns and Hirt 1987; Johns 1993).

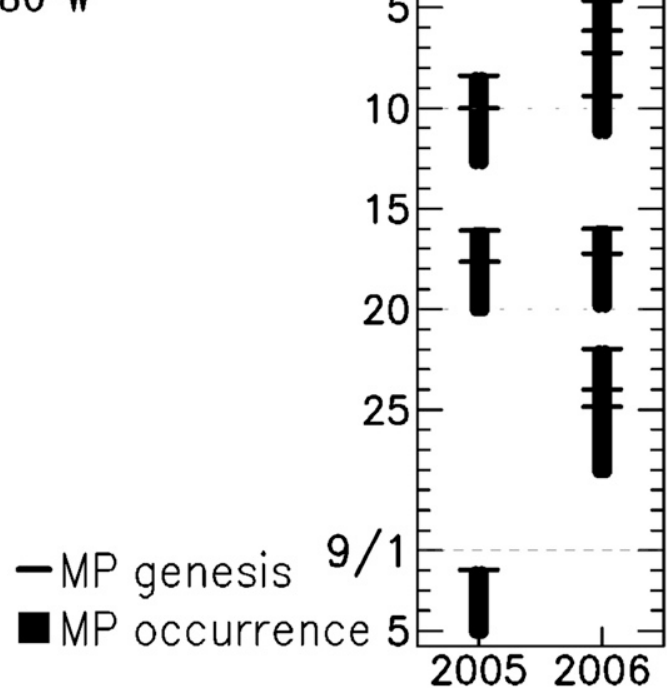

A snapshot of the NAM05 forecast on this particular day is displayed in Fig. 2b at forecast hour 21 (initialized at 1200 UTC 20 July). Overall, the background flows at all levels are consistent with the observed. The $600-\mathrm{hPa}$ streamlines also depict the perturbations (marked by solid lines) near the precipitation areas, but the forecasted MPs 1 and 2 appear to fall behind the observed ones. The forecasted precipitation pattern is very different from that of stage IV, including the displaced storm rainfall associated with the MPs and the false rainband across Illinois and Iowa. Moreover, precipitation amounts over the storm area are apparently undersimulated. Another example MP on 5 August 2006 is shown in Fig. 3a. The performance of NAM06 on this MP 
TABLE 1. Basic parameters of MPs analyzed in this study, including the date when the MP is within the detection domain $\left(35^{\circ}-47^{\circ} \mathrm{N}, 100^{\circ}-85^{\circ} \mathrm{W}\right)$, the maximum $\zeta$ after a nine-point smoothing, the maximum diameter measured by the zonal distance of the positive $\zeta$ at $600 \mathrm{hPa}$, and the estimated zonal distance of the maximum precipitation associated with the MP within the $1 \mathrm{~mm} \mathrm{~h}^{-1}$ contours. MPs not associated with convection are noted as "no convection."

\begin{tabular}{|c|c|c|c|}
\hline Date & $\begin{array}{c}\operatorname{Max} \zeta \\
\left(10^{-5} \mathrm{~s}^{-1}\right)\end{array}$ & $\begin{array}{l}\text { Diameter of } \\
\text { MPs }(\mathrm{km})\end{array}$ & $\begin{array}{c}\text { Precipitation } \\
\text { boundary }(\mathrm{km})\end{array}$ \\
\hline 2 Jul 2005 & 5.8 & 550 & 120 \\
\hline 8 Jul 2005 & 5.4 & 550 & 150 \\
\hline 12 Jul 2005 & 3.1 & 600 & 100 \\
\hline $20 \mathrm{Jul} 2005$ & 5.2 & 750 & 220 \\
\hline $21 \mathrm{Jul} 2005$ & 7.4 & 800 & 250 \\
\hline $22 \mathrm{Jul} 2005$ & 6.0 & 650 & 80 \\
\hline $23 \mathrm{Jul} 2005$ & 3.6 & 550 & 150 \\
\hline $24 \mathrm{Jul} 2005$ & 9.5 & 850 & 120 \\
\hline 29 Jul 2005 & 7.1 & 700 & 70 \\
\hline $30 \mathrm{Jul} 2005$ & 5.2 & 650 & No convection \\
\hline 31 Jul 2005 & 9.0 & 850 & 110 \\
\hline 1 Aug 2005 & 2.3 & 900 & No convection \\
\hline 9 Aug 2005 & 6.0 & 550 & 130 \\
\hline 11 Aug 2005 & 4.7 & 1100 & 300 \\
\hline 12 Aug 2005 & 6.5 & 1000 & 240 \\
\hline $1 \mathrm{Jul} 2006$ & 4.3 & 1050 & 250 \\
\hline 2 Jul 2006 & 4.5 & 950 & 240 \\
\hline 3 Jul 2006 & 5.1 & 800 & 150 \\
\hline 4 Jul 2006 & 4.9 & 700 & 170 \\
\hline 6 Jul 2006 & 4.7 & 700 & No convection \\
\hline 8 Jul 2006 & 9.3 & 750 & 120 \\
\hline $10 \mathrm{Jul} 2006$ & 5.4 & 1100 & 270 \\
\hline 11 Jul 2006 & 8.2 & 1150 & 310 \\
\hline 14 Jul 2006 & 6.0 & 850 & 180 \\
\hline 19 Jul 2006 & 9.5 & 600 & 160 \\
\hline $20 \mathrm{Jul} 2006$ & 3.6 & 800 & 140 \\
\hline $25 \mathrm{Jul} 2006$ & 4.3 & 650 & 80 \\
\hline $26 \mathrm{Jul} 2006$ & 6.5 & 700 & No convection \\
\hline 4 Aug 2006 & 7.2 & 700 & No convection \\
\hline 5 Aug 2006 & 10.1 & 650 & 270 \\
\hline 6 Aug 2006 & 3.7 & 800 & 210 \\
\hline 8 Aug 2006 & 2.8 & 900 & 330 \\
\hline 10 Aug 2006 & 5.8 & 1050 & 290 \\
\hline 11 Aug 2006 & 3.0 & 550 & 120 \\
\hline 23 Aug 2006 & 6.9 & 700 & 160 \\
\hline
\end{tabular}

event (Fig. 3b) reveals similar deficiencies as in NAM05: westward displacement of the MP position associated with displaced and underpredicted precipitation.

Around 1200 UTC on 21 July 2005, the derecho MCS associated with MP 2 produced an MCV in the trailing stratiform region (Fig. 4a), as indicated by the comma-shaped, rotating structure of the composite radar reflectivity (cf. Olsson and Cotton 1997) near the Minnesota-Iowa border. The MCV occurred slightly to the east of MP 2's vorticity center, and continued to grow and move eastward, while the MP propagated southeastward (Fig. 4b). The MCV and its parent MCS, both maintaining an eastward course, eventually separated from the southeastward-moving MP by 0000 UTC 22 July (not shown). The increasing relative vorticity of the MP during this time frame suggests that the MCV formation simultaneously amplifies MP 2. MCVs forming within the stratiform region behind line convection are a wellknown phenomenon that is still studied today (Davis and Trier 2007; Schumacher and Johnson 2008; Galarneau et al. 2009). It is known that MCVs are produced primarily by diabatic heating with little external forcing (e.g., Hertenstein and Schubert 1991), though interactions between MCVs and MPs (i.e., preexisting vorticity source in northwest flow) are not well documented. The incorrectly forecasted MCS associated with MP 2 (Fig. 2b) indicates that the MCV (Fig. 4) was either not properly simulated or not simulated at all. The implication of such a bias on MP forecasting will be discussed further in section 4c.

The evolution of the MP series during 20-22 July 2005 is displayed by the Hovmöller diagram of the observed relative vorticity at $600 \mathrm{hPa}$ (Fig. 5a), along with three NAM05 forecasts initialized at 1200 UTC 19 July, 0600 UTC 20 July, and 0000 UTC 21 July in Figs. 5b-d, respectively. NAM05 depicts MP 1 relatively well (Fig. 5b), despite many smaller-scale differences, but MPs 2 and 3 are too disorganized to be considered valid predictions. The forecast of MP 2 is improved when the model is initialized near the MP genesis time (Fig. 5c), although the MP appears too weak and its life cycle too short. Even when initialized "in progress" (Fig. 5d), the life cycle of forecasted MP 2 remains too short with the vorticity structure distorted. The increasing deviations between the forecasted vorticity and observed MP tracks in Figs. $5 \mathrm{~b}-\mathrm{d}$ indicate that the propagation speeds of the forecasted MPs are systematically slower than that observed. Such a slower-speed bias is reflected in the forecasted precipitation in Fig. 6 . The precipitation forecasts in the first two runs (Figs. $6 \mathrm{~b}$ and $6 \mathrm{c}$ ) are particularly poor, with the two rainbands severely distorted near $90^{\circ} \mathrm{W}$.

Note that the rapid amplification of MP 2 around 1200 UTC 21 July (Fig. 5a) coincides with the MCV development as shown in Fig. 4, but the first two runs of the forecast fail to predict such amplification (Figs. 5b and 5c). This error corresponds to the ill-simulated precipitation (and convection) that may link to an ill-simulated MCV generation. The MP amplification is forecasted in the third run (Fig. 5d), but it remains too slow relative to the observed. Similar analysis of the 5 August 2006 MP case (not shown) reveals the same deficiencies of slow propagation and displaced precipitation, though no MCV is observed near or within this MP. These biases indicate that the causes of the NAM's difficulty in simulating progressive MCSs are manifold. Nevertheless, the above analysis highlights two systematic biases of MP forecasting: 1) propagation speed and 2) precipitation location and 
$(\mathrm{a})(\mathrm{V}, \mathrm{P})(\mathrm{Obs})$
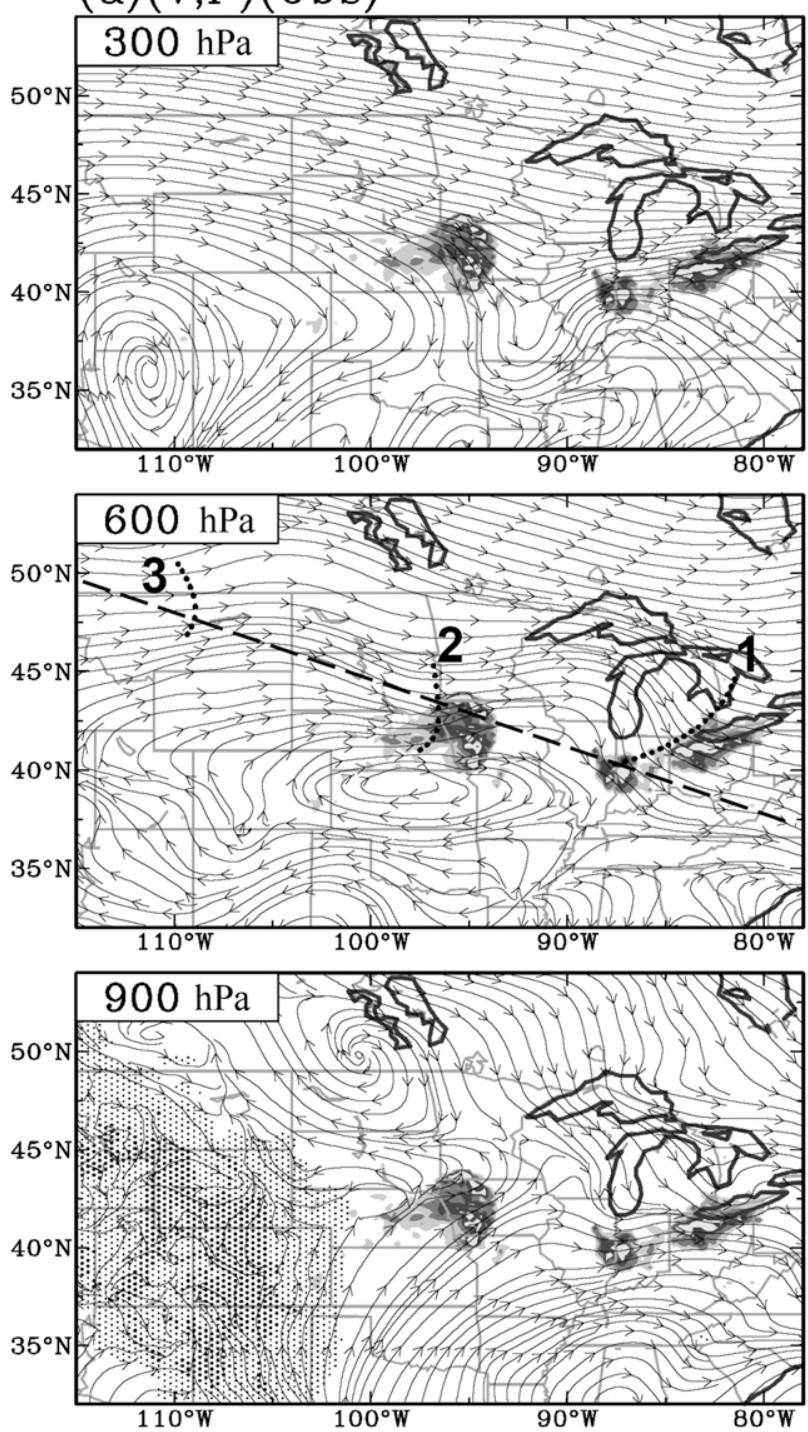

(b) $(\mathrm{V}, \mathrm{P})(\mathrm{NAM}-\mathrm{fst})$
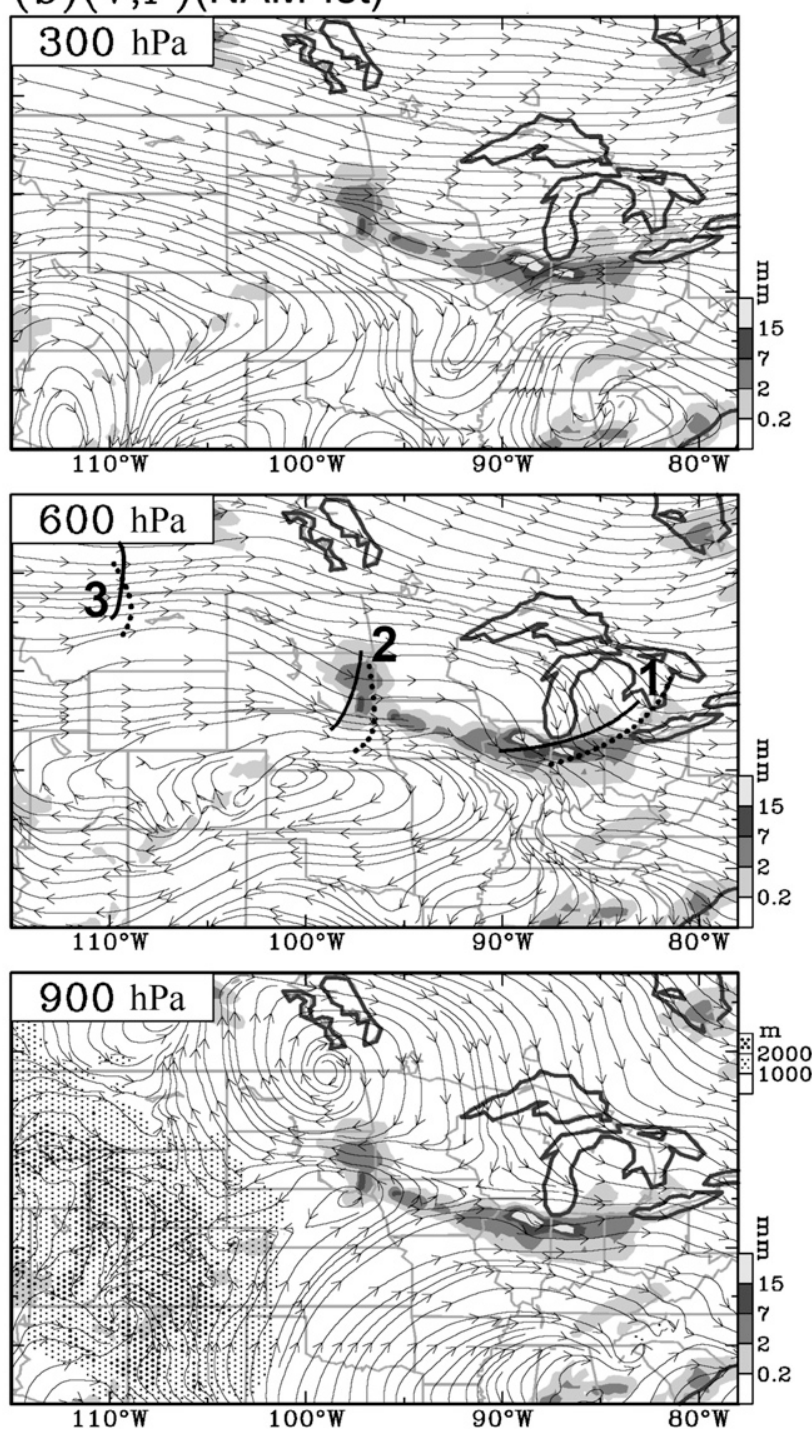

FIG. 2. (a) The NARR streamlines (V) at (top to bottom) 300, 600, and $900 \mathrm{hPa}$ at 0900 UTC 21 Jul 2005 superimposed with stage IV precipitation ( $P$; 3-h accumulation). (b) As in (a) but for the NAM forecast initialized at 1200 UTC 20 Jul. The observed MP troughs are marked by dashed lines at $600 \mathrm{hPa}$ with the corresponding case number, while the forecasted MP troughs are marked by solid lines in (b). Terrain is indicated by dotted areas at $900 \mathrm{mb}$.

intensity. These two biases will be examined in the next section.

\section{Diagnostic analyses on the forecast of MPs}

The NAM performs well in capturing the geneses of all 25 MPs at forecast hour 0. Therefore, forecasted MPs are defined as those initialized at the genesis time of each perturbation. ${ }^{1}$ This approach sets the beginning of

\footnotetext{
${ }^{1}$ MP geneses occurring at 0300, 0900, 1500, and 2100 UTC are categorized into forecasts initialized at 0600, 1200, 1800, and 0000 UTC, respectively.
}

each MP at hour 0 , so the evolutions of the observed and forecasted MPs can be synchronized and presented in terms of forecast hour.

\section{a. Propagation errors of MPs}

The position errors of forecasted MPs are obtained by calculating the instantaneous longitudinal and latitudinal location differences between forecasted and observed MPs at each 6-h cycle. The mean longitudinal (latitudinal) position errors during the life cycles of MPs are shown in Fig. 7a (Fig. 7b). The genesis locations of MPs are handled relatively well by the NAM's initial 

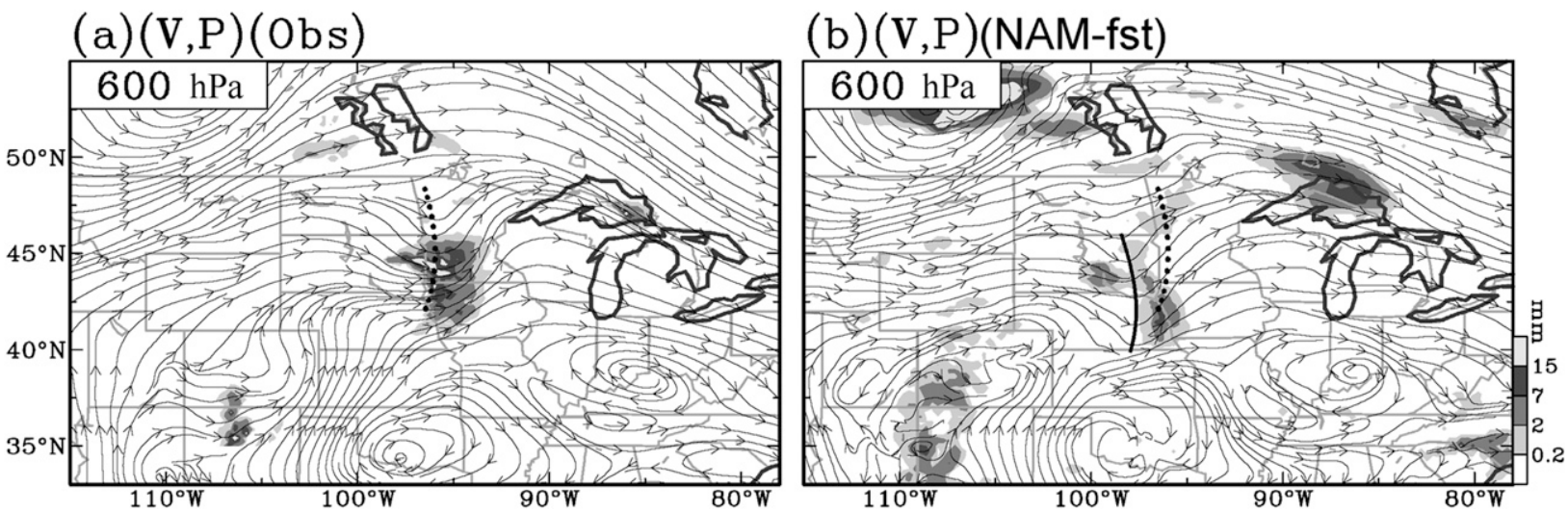

FIG. 3. As in Fig. 2, but at (a) 0900 UTC 5 Aug 2006 at $600 \mathrm{hPa}$ in and (b) for the NAM forecast initialized at 1200 UTC 4 Aug 2006.

analysis, especially by NAM05, as indicated by small differences at the zero hour. These small differences may simply exist because the NAM05 and NARR are basically the Eta Model. Nonetheless, the increasing distance between forecasted and observed MPs in Fig. 7a indicates that forecasted MPs constantly lag the observed ones. NAM06 exhibits similarly systematic, but less severe, position errors after hour 24. A southward deviation of MPs in NAM05 becomes more pronounced during hours 24-36 but appears to be overcorrected at hour 48 (Fig. 7b). NAM06 also reduces this meridional position error. The speed error (Fig. 7c), computed from the MPs' propagation speed difference at each 6-h interval, mainly reflects the zonal position errors and shows that forecasted MPs are systematically too slow and decelerating.

In the dynamic perspective, the propagation of a cyclonic circulation is governed by the vorticity tendency $\left(\zeta_{t}\right)$ determined in the vorticity budget equation. Analyzing the formation mechanism of MPs, our recent study (Wang et al. 2009b, manuscript submitted to Climate Dyn.) showed that horizontal vorticity advection is the major forcing in the vorticity budget propagating the perturbations. The $\sim 700 \mathrm{~km}$ diameter of MPs leads the horizontal scale of these perturbations to be closer to the synoptic scale $\left(10^{3} \mathrm{~km}\right)$ than the mesoscale $\left(10^{2} \mathrm{~km}\right)$. Using $L \sim 10^{3} \mathrm{~km}$, the vorticity budget equation can be scaled to

$$
\zeta_{t}\left(=\frac{\partial \zeta}{\partial t}\right) \approx-\mathbf{V} \cdot \nabla \zeta-v \beta-f \nabla \cdot \mathbf{V}-\zeta \nabla \cdot \mathbf{V},
$$

with conventional symbols (Holton 2004). All terms of Eq. (1) are averaged from the 25 cases at hour 24 and shown in Fig. 8a based on the NARR. Each term is an average within a $4^{\circ} \times 4^{\circ}$ area centered at the maximum $\zeta_{t}$ associated with MPs, which is always distributed im- mediately east of the perturbations. Despite the dominant contribution of the relative vorticity advection $(87 \%)$ to $\zeta_{t}$, the total vortex stretching $[-(\zeta+f) \boldsymbol{\nabla} \cdot \mathbf{V}]$ contributes $15 \%$ with the majority $(13 \%)$ formed by $-f \nabla \cdot \mathbf{V}$. This reflects the fact that vortex stretching is generally small in the midtroposphere but is not negligible, particularly when convection is present (e.g., Zhang 1992). Planetary vorticity advection $(-v \beta)$ is unimportant due to small changes in $\beta$ relative to the scale of the MPs. The tilting term $[(\partial \omega / \partial y)(\partial v / \partial p)-$ $(\partial \omega / \partial x)(\partial u / \partial p)]$ is also small. Therefore, for MPs Eq. (1) can be approximated as

$$
\zeta_{t} \approx-\mathbf{V} \cdot \nabla \zeta-f \nabla \cdot \mathbf{V},
$$

with $-\mathbf{V} \cdot \nabla \zeta$ being a more prominent forcing than $-f \nabla \cdot \mathbf{V}$ in the propagation stage. The NAM biases of the vorticity budget are shown in Fig. 8b. Relative vorticity advection becomes even more dominant (97\%) in the contribution of the $\zeta_{t}$ bias compared to vortex stretching $(8 \%)$.

The ambient flow speed and relative vorticity gradient determine $-\mathbf{V} \cdot \nabla \zeta$. To examine their contributions in the $-\mathbf{V} \cdot \nabla \zeta$ bias, wind speeds of the NAM and the NARR at $600 \mathrm{hPa}$ averaged over a $4^{\circ} \times 4^{\circ}$ domain centered at each MP are compared in Fig. 9. There is a minor bias toward large wind speeds, indicating an underpredicted environmental flow speed. This bias becomes significant when the observed winds are stronger than $15 \mathrm{~m} \mathrm{~s}^{-1}$. Applying the same procedure in Fig. 7 to each 6-h position of MPs, the 600-hPa $\zeta$ evolutions of simulated and observed MPs in 2005 and 2006 are shown in Figs. 10a and 10c, respectively. A rapid growth of the perturbation vorticity occurs during hours 18-24, a time frame when MPs usually move into the Upper Midwest (WCC) while the associated convection is developing (cf. Fig. 6). The intensity of $\zeta$ is constantly undersimulated 

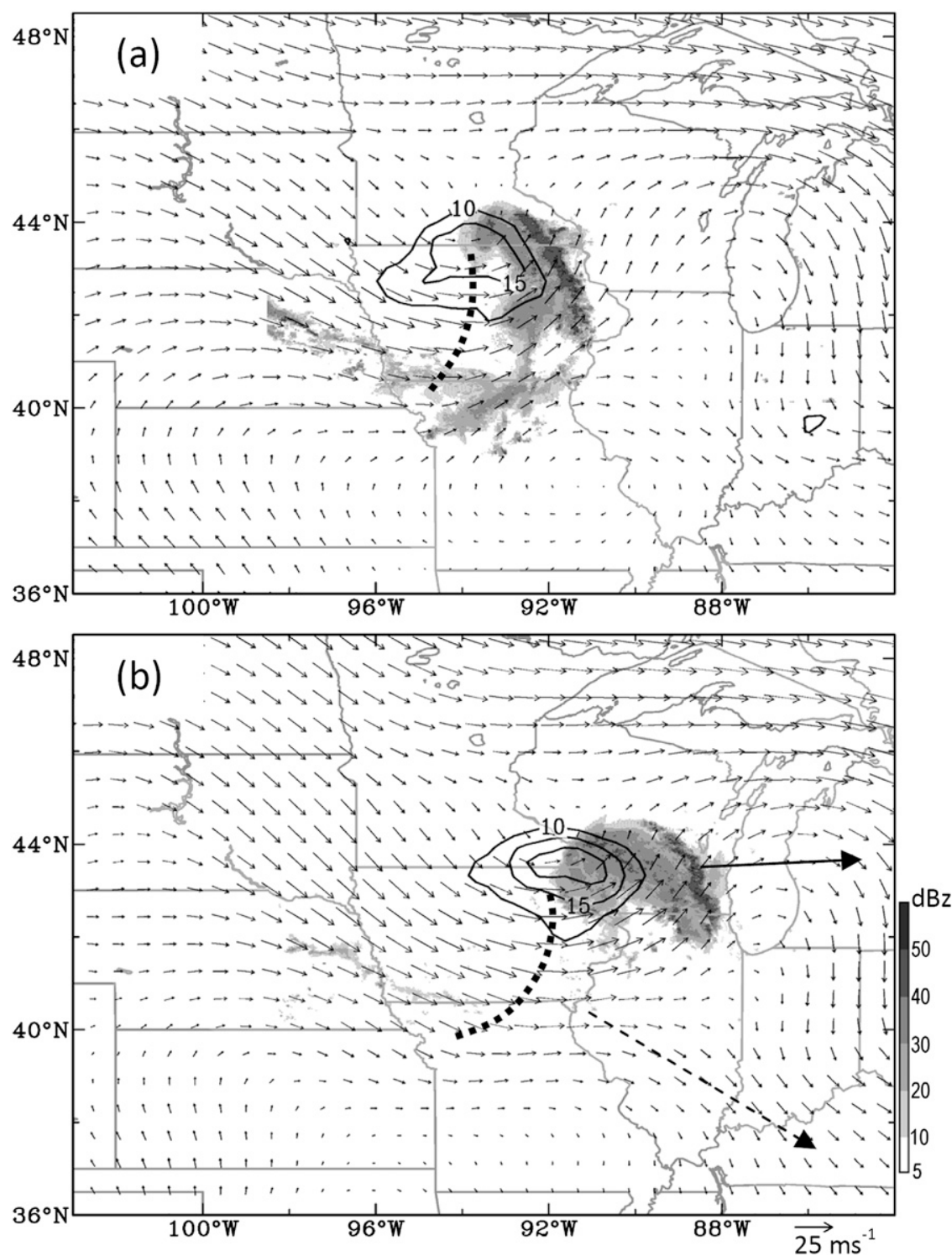

FIG. 4. Composite radar reflectivities superimposed with $600-\mathrm{hPa}$ wind vectors and relative vorticity (contour interval $5 \times 10^{-5} \mathrm{~s}^{-1}$ starting from $10 \times 10^{-5} \mathrm{~s}^{-1}$ ) on $21 \mathrm{Jul} 2005$ at (a) 1200 and (b) 1500 UTC. The trough line of the MP is marked by thick dashed lines. The future movement of the MCV (MP) is indicated by a solid (dashed) arrow line.

(Figs. 10b and 10d), though such a bias is reduced by a half in NAM06. Comparing Figs. 9 and 10, it appears that the speed bias of the MPs is related to the perturbation vorticity and ambient flow speed, which are both underestimated.

These observations are examined through a reconstruction of the MP propagation speed using the vorticity budget equation. The propagation speed of a cyclonic perturbation can be estimated through $\zeta_{t}$ :

$$
c_{\zeta} \equiv \frac{\Delta L \zeta_{t}}{\zeta}
$$

where $\Delta L$ is the distance a perturbation travels during the time interval in which $\zeta_{t}$ is calculated. According to Eq. (2), $c_{\zeta}$ can be approximated as

$$
c_{\zeta} \approx \frac{\Delta L(-\mathbf{V} \cdot \nabla \zeta-f \nabla \cdot \mathbf{V})}{\zeta}
$$

Based on the dominance of $-\mathbf{V} \cdot \nabla \zeta$ in the bias (Fig. 8b), Eq. (4a) can be further simplified as

$$
c_{\zeta} \approx \frac{\Delta L(-\mathbf{V} \cdot \nabla \zeta)}{\zeta}
$$




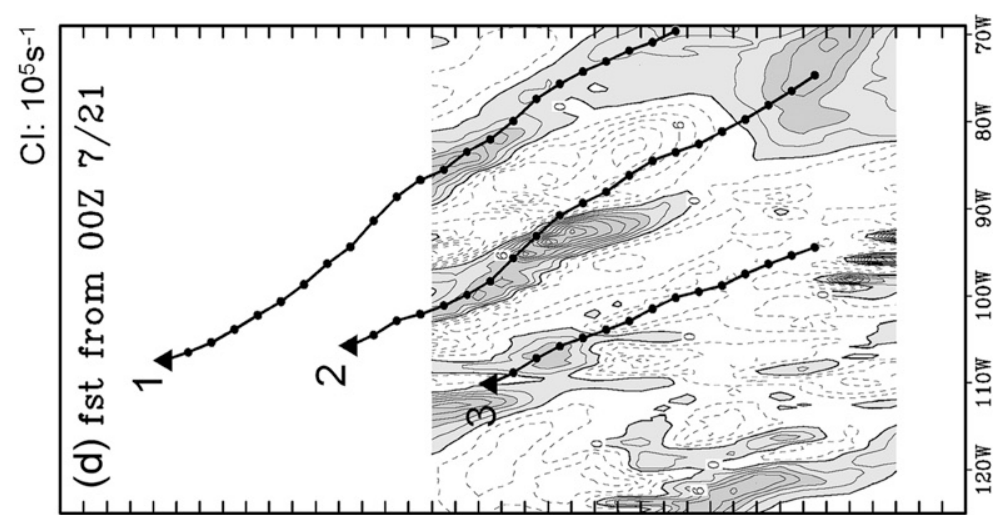

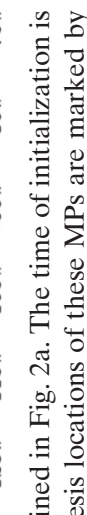

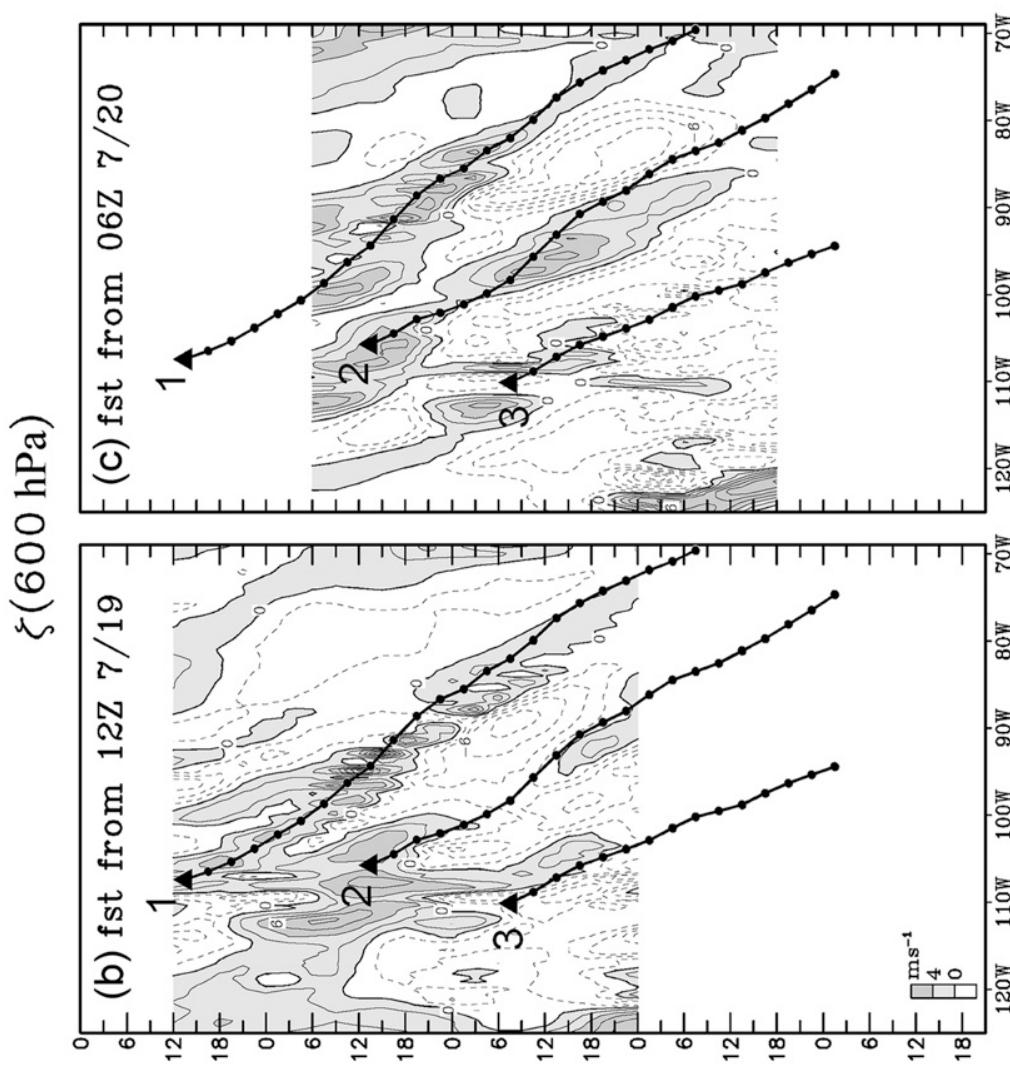

응

全守

官

क

\% ฐ ฮ

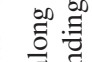

西

दे के

응

ส

兽节

.

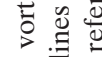

ग्ञ

ठु 융

는 을

- 它

월

1 .

르를

ฮ

गुำ

D

สิ

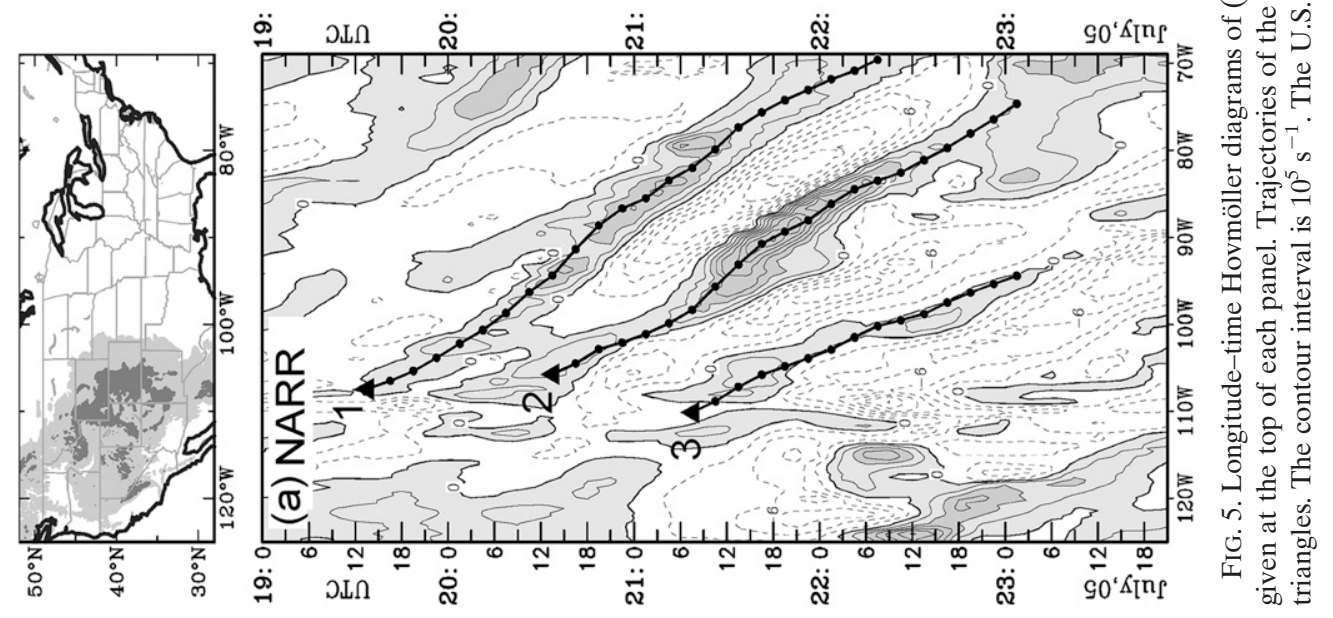



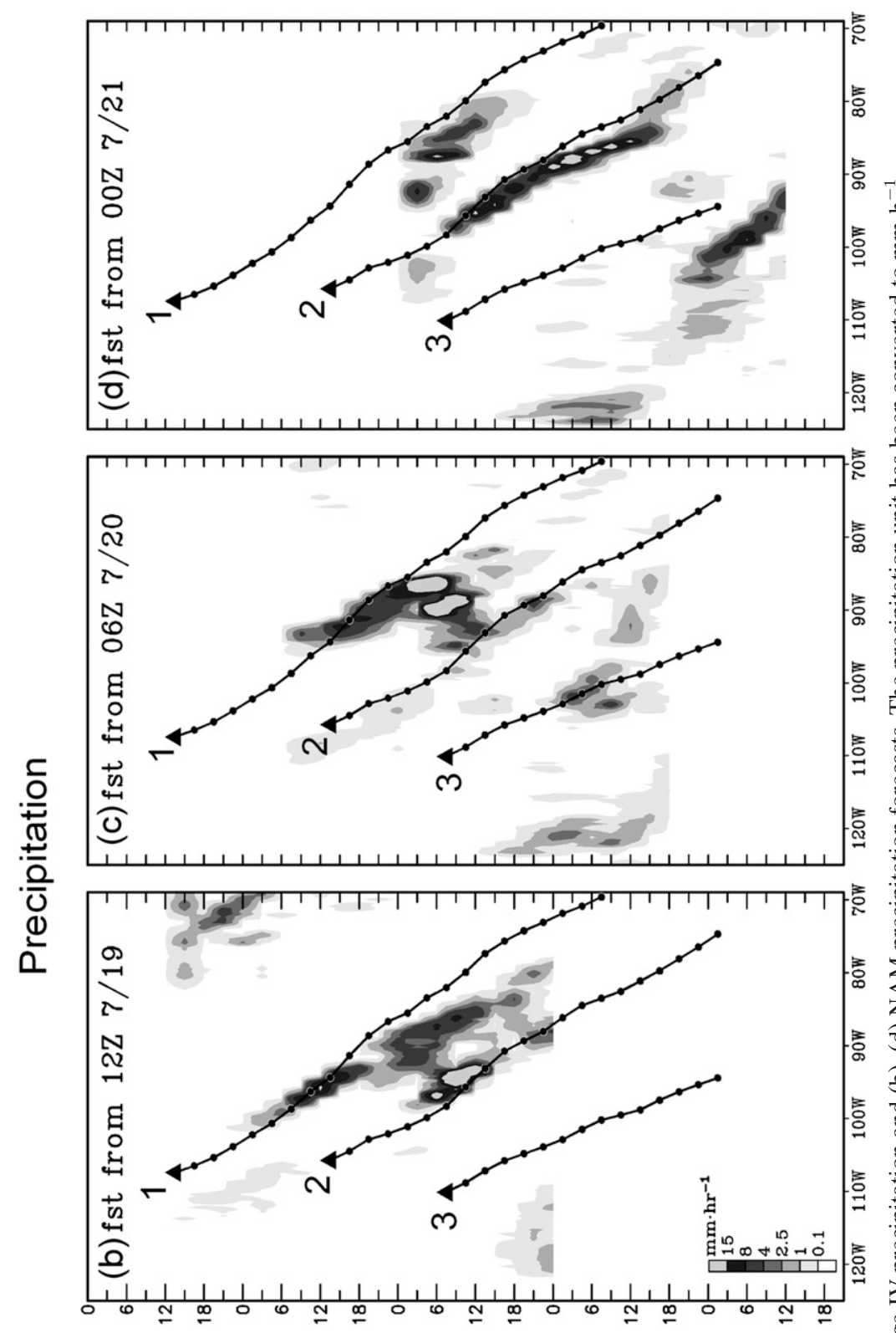

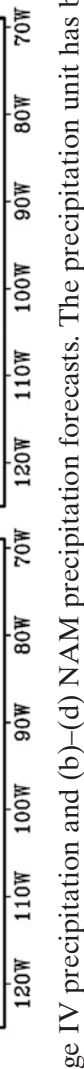
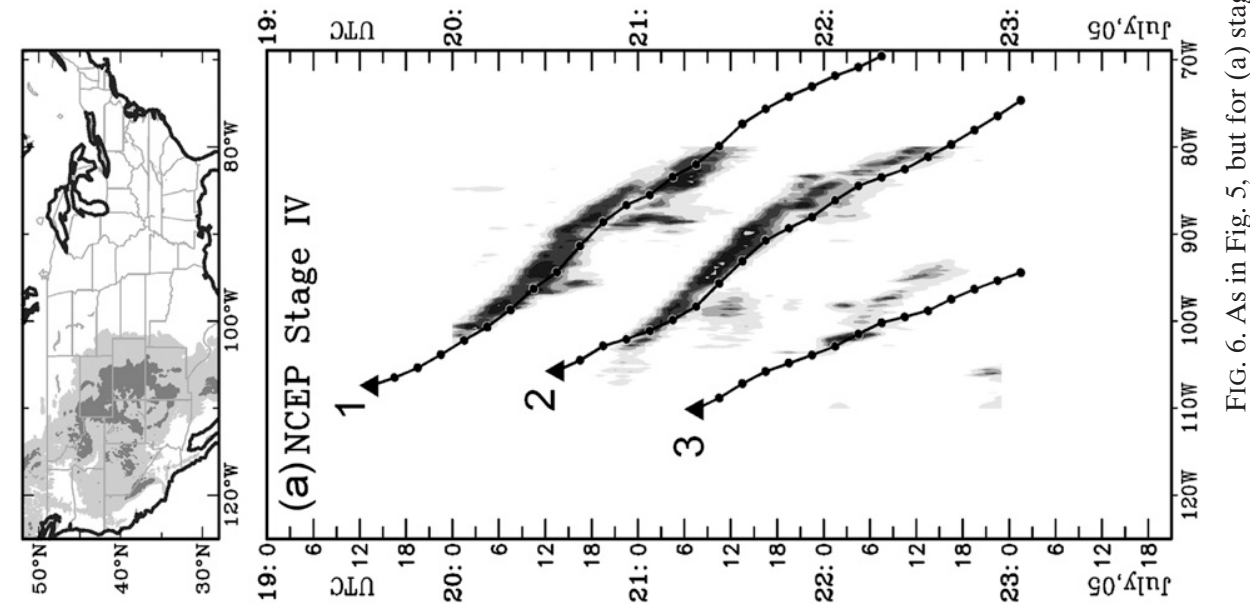

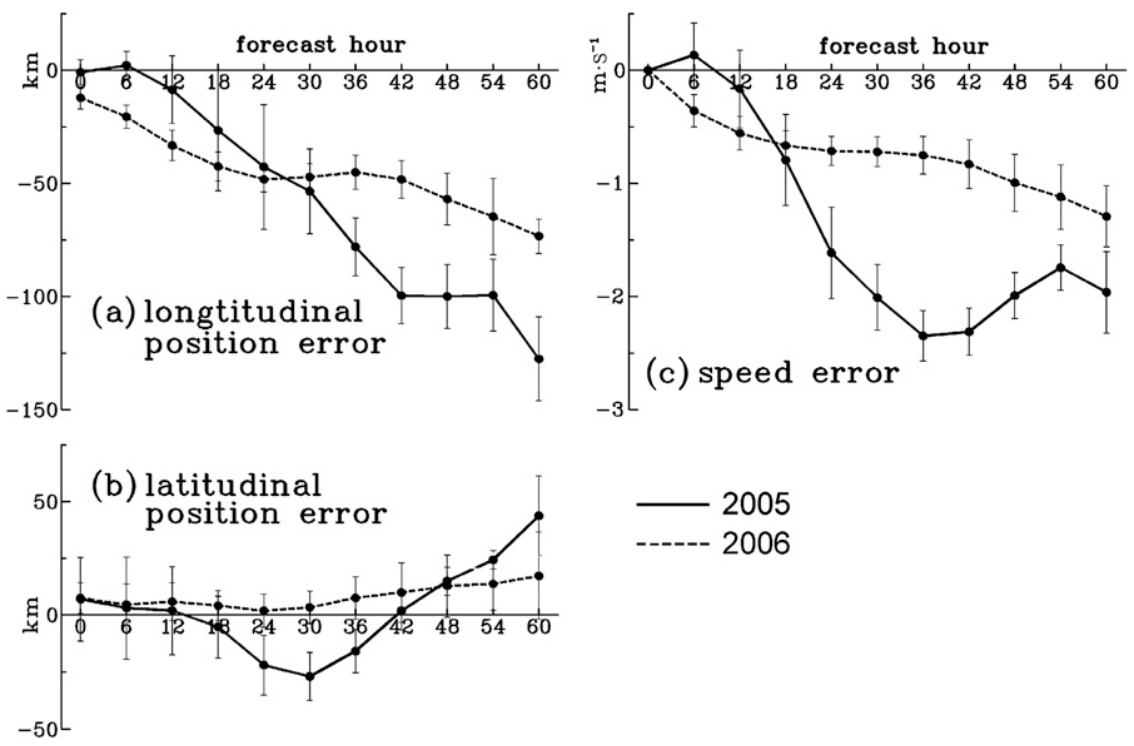

FIG. 7. Mean position errors of forecasted MPs in the (a) longitudinal and (b) latitudinal directions, and (c) the mean speed errors of the forecasted MPs during a 60-h life cycle. The range of one standard deviation (error bar) is shown by thin lines. Solid lines indicate 2005 and gray dashed lines represent 2006. Error bars are given every 6 h.

Equation (4b) is obtained through the following approach: $\zeta$ and $-\mathbf{V} \cdot \nabla \zeta$ are averaged over a $4^{\circ} \times 4^{\circ}$ domain centered at the maximum of $\zeta_{t}$, and $\Delta L$ is determined by the distance between the previous $3-\mathrm{h}$ and the next 3-h positions of the MP. Here, the observed $\Delta L$ is used for forecasted MPs for an easy evaluation of different vorticity forcing terms. ${ }^{2}$ Such a diagnostic approach has been used to estimate the propagation speed of simulated tropical cyclones (e.g., Carr and Elsberry 1995). Because the biases in propagation speed and vorticity are more significant in 2005 than 2006, we only present the results of NAM05.

Shown in Fig. 11a is $c_{\zeta}$ of the NARR versus the actual speeds of MPs during hours 24-30 with a 3-h interval. In spite of the deviations between the estimated and actual speeds of individual MPs, the concentration of $c_{\zeta}$ near the center line (with a correlation coefficient of 0.83 ) shows that $-\mathbf{V} \cdot \nabla \zeta$ provides a good approximation for the propagation speed of the MPs. Note that the mean speed of $c_{\zeta}\left(\sim 18 \mathrm{~m} \mathrm{~s}^{-1}\right)$ is near that of summer convective episodes (Carbone et al. 2002; Davis et al. 2003; Trier et al. 2006) and progressive derechos (Johns and Hirt 1987). Plotting $c_{\zeta}$ of the NARR versus that of NAM05 (Fig. 11b) shows that $c_{\zeta}$ of NAM05 shifts toward

\footnotetext{
${ }^{2}$ Using $\Delta L$ in the NAM results in an additional $5 \%$ of bias in $c_{\zeta}$ toward slower speed. The lack of this bias does not affect the diagnosis of Fig. 11 with regard to the contributions of vorticity forcing to the vorticity tendency.
}

the NARR, suggesting that the forecasted $c_{\zeta}$ is systematically too slow in responding to weak $-\mathbf{V} \cdot \nabla \zeta$. To assess the contributions of the relative vorticity and wind speed to this $-\mathbf{V} \cdot \nabla \zeta$ bias, the structural difference between the forecasted and observed MPs needs to be examined.

An MP-related composite analysis used in WCC is adopted here. Because the instantaneous location of each MP is different from any other, realignment is performed in the composite analysis. The mean position of MPs during hour 24 appears to be near the border between Iowa and Nebraska; thus, each MP center is shifted to this mean position for the composite. The 2005 cases used for the composite are listed in Table 1. Composite $600-\mathrm{hPa}$ streamlines of the NARR and NAM05 are shown in Figs. 12a and 12d, respectively, superimposed with relative vorticity advection. The intensity of the forecasted MP and its relative vorticity advection are apparently weaker than the observed. A Fourier wave decomposition is then applied to separate the perturbation from the ambient flows. The MP is isolated using the short-wave regime of zonal wavenumbers $\geq 10$ (globally) based on the $\sim 700 \mathrm{~km}$ diameter of MPs, as shown by the filtered short-wave streamlines and $\zeta$ in Figs. 12b and 12e. The forecasted perturbation and vorticity (Fig. 12b) are less organized and weaker than the observed (Fig. 12e). On the other hand, flows in the long-wave regime (zonal wavenumbers 0-9; Figs. 12c and 12f) do not show noticeable differences between NAM05 and the NARR, suggesting that environmental wind speed is not as crucial as perturbation vorticity. 

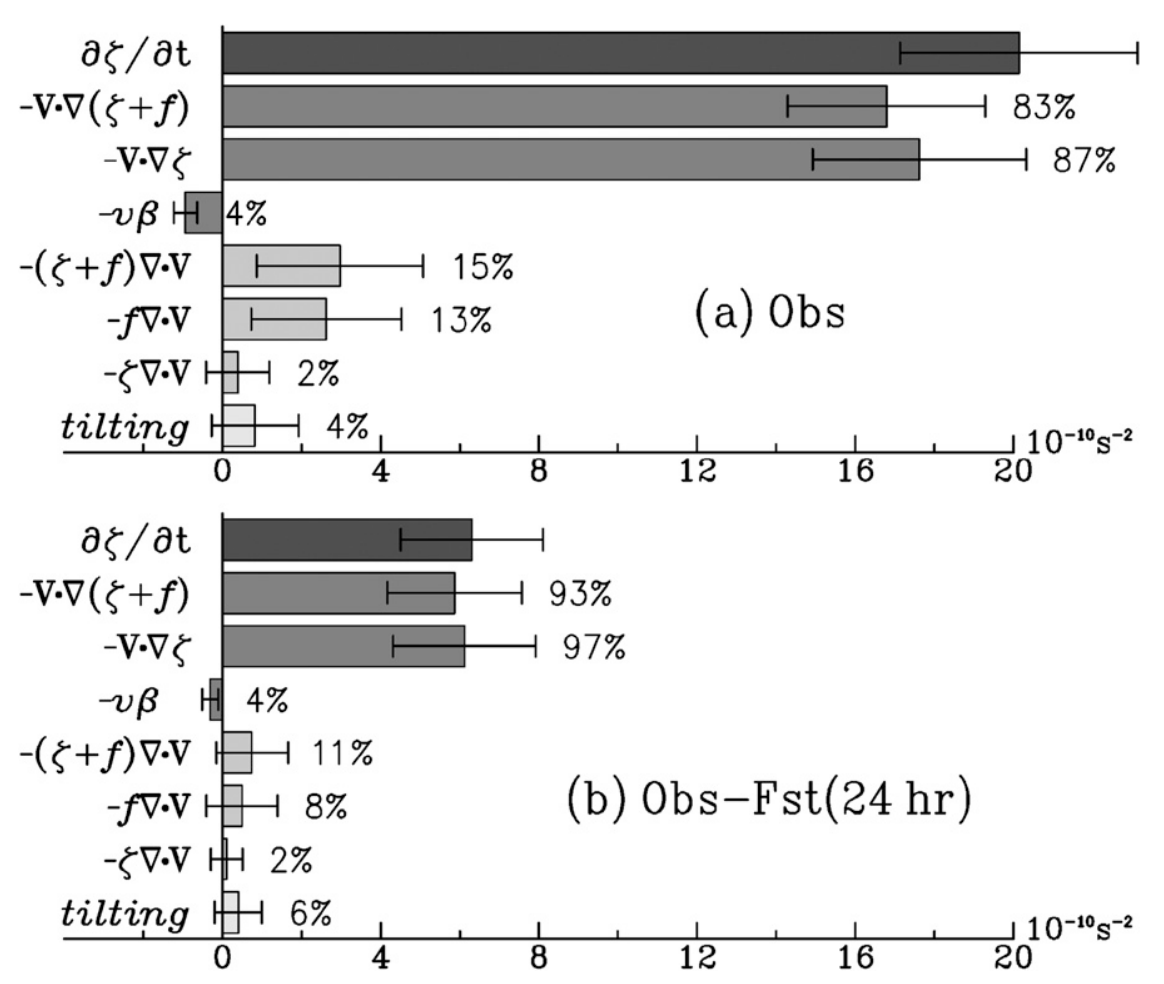

FIG. 8. (a) Vorticity budget terms of MPs [Eq. (1)] at hour 24 based on the NARR averaged over a $4^{\circ} \times 4^{\circ}$ domain centered at the maximum vorticity tendency at $600 \mathrm{hPa}$. (b) As in (a) but for the differences between the observation and the NAM forecast. Error bars are added in each term. Note that the tilting term is not included in Eq. (1).

After the wave filtering, a diagnostically "improved" MP flow pattern can be reconstructed by combining the observed short-wave perturbation (Fig. 12e) with the forecasted background flow (Fig. 12c). The reconstructed relative vorticity advection is expressed as

$$
-\mathbf{V}^{*} \cdot \nabla \zeta^{*}=-\left(\mathbf{V}_{\mathrm{NARR}}^{S}+\mathbf{V}_{\mathrm{NAM}}^{L}\right) \cdot \nabla\left(\zeta_{\mathrm{NARR}}^{S}+\zeta_{\mathrm{NAM}}^{L}\right)
$$

where $(\cdot)^{S}$ and $(\cdot)^{L}$ denote the short- and long-wave regimes, respectively. Results are shown in Fig. 12g. The reconstructed MP structure is very similar to the observations (Fig. 12d), and $-\mathbf{V}^{*} \cdot \nabla \zeta^{*}$ is visually identical to the observed $-\mathbf{V} \cdot \nabla \zeta$. The values of reconstructed $c_{\zeta}^{*}$ during hours 24-30 (Fig. 11c) become close to the observed propagation speed. Repeated tests on NAM06 yield consistent (but smaller) biases with NAM05 (not shown). To examine the bias of strong environmental winds (Fig. 9) on $c_{\zeta}^{*}$, the group of cases with the observed wind speed larger than $15 \mathrm{~m} \mathrm{~s}^{-1}$ and the forecasted wind speed smaller than the observed are highlighted in Fig. 11c by open circles. As indicated by the fitted line of this group of cases, there remains a $\sim 10 \%$ speed bias from the observed $c_{\zeta}$. This additional bias may even increase when integrated over time. For the rest of the MPs, the effect of ambient flow speed on the $c_{\zeta}^{*}$ bias remains insignificant $(<2 \%$ on average).

It is observed in Figs. 7 and 10 that MPs undergo noticeable acceleration and amplification between hours 18 and 24 when the associated convection is developing. Organized convection often leads to vorticity generation through vortex stretching (Zhang 1992) and/or heating (Hertenstein and Schubert 1991). During the convective development stage at hours 18-21, it is found that $c_{\zeta}^{*}$ based on $-\mathbf{V} \cdot \nabla \zeta$ (Fig. 11d) is insufficient to compensate the propagation speed bias, as there exists a $\sim 15 \%$ bias. To remedy this, $c_{\zeta}^{*}$ is reconstructed using Eq. (4a) by adding vortex stretching as $-f \nabla \cdot\left(\mathbf{V}_{\mathrm{NARR}}^{S}+\mathbf{V}_{\mathrm{NAM}}^{L}\right)$ and shown in Fig. 11e. Visual inspection finds that the remaining bias in Fig. 11d is largely eliminated, indicating that the effects of $-f \nabla \cdot \mathbf{V}$ cannot be neglected.

The circulation differences of the MP composites between NAM05 and the NARR (Fig. 13a) depict positive streamfunction anomalies and anticyclonic flows over the western and northern United States. The anomalous anticyclone extends to lower levels (Fig. 13b) and creates easterly anomalies across the Midwest. It appears that the midsummer anticyclone (Fig. 1a) is too strongly simulated by NAM05, while the MP is embedded in 


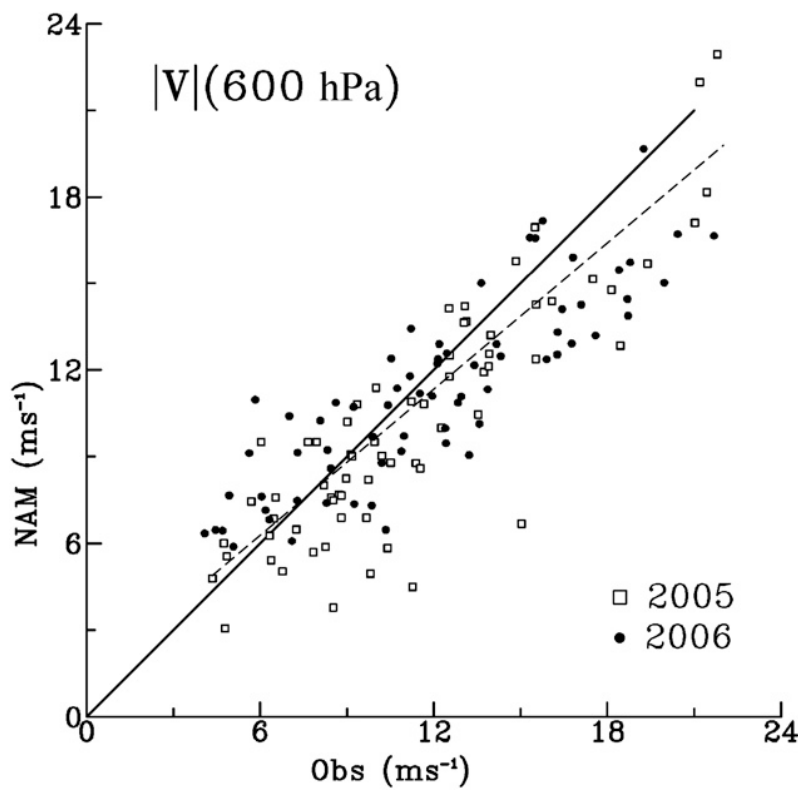

FIG. 9. NAM-forecasted wind speed ( $y$ axis) at $600 \mathrm{hPa}$ vs that observed ( $x$ axis), averaged over a $4^{\circ} \times 4^{\circ}$ area over each MP at hour 24. Values during 2005 (2006) are marked by open rectangles (solid dots). A linear-fitted line (dashed line) is added for all values.

these anticyclonic and easterly biases. Because the vertical development of the MPs is suppressed by the upperlevel monsoon anticyclone (WCC), it is feasible that the anticyclonic bias similarly reduces the strength of the perturbation vorticity. The easterly anomalies sur- rounding the MP correspond to the bias of the weak ambient flow that also slows down the perturbation. In addition, the anomalous northerly flow over the Great Plains (Fig. 13b) implies that the simulated low-level jet is too weak.

\section{b. Precipitation errors of MPs}

Forecasted precipitation associated with MPs is evaluated through equitable threat scores (ETSs; Schaefer 1990) and bias scores, following the formulation in Jankov and Gallus (2004). Conventionally, ETSs are computed within a fixed domain. Because MPs are propagating systems with a relatively small dimension, it is improper to use a large, fixed domain that may include rainfall generated by irrelevant systems (McBride and Ebert 2000). Thus, a system-following verification approach is a more effective way of evaluating QPFs associated with MPs. A modified ETS is designed for this purpose: scores are calculated within a $15^{\circ} \times 15^{\circ}$ domain centered at each observed MP every $6 \mathrm{~h}$. Since each ETS domain at a given time is different from any other, this procedure creates "mobile" ETSs and bias scores. The setting of rainfall thresholds for ETSs and bias scores follows that used in Jankov and Gallus (2004) and Clark et al. (2007).

Average ETSs and biases calculated from the above procedure at every 6-h period (not shown) exhibit the highest scores at forecast hour 12 during both 2005 (Fig. 14a) and 2006 (Fig. 14b). Forecast hour 12 is generally when the mesoscale portion of the kinetic energy
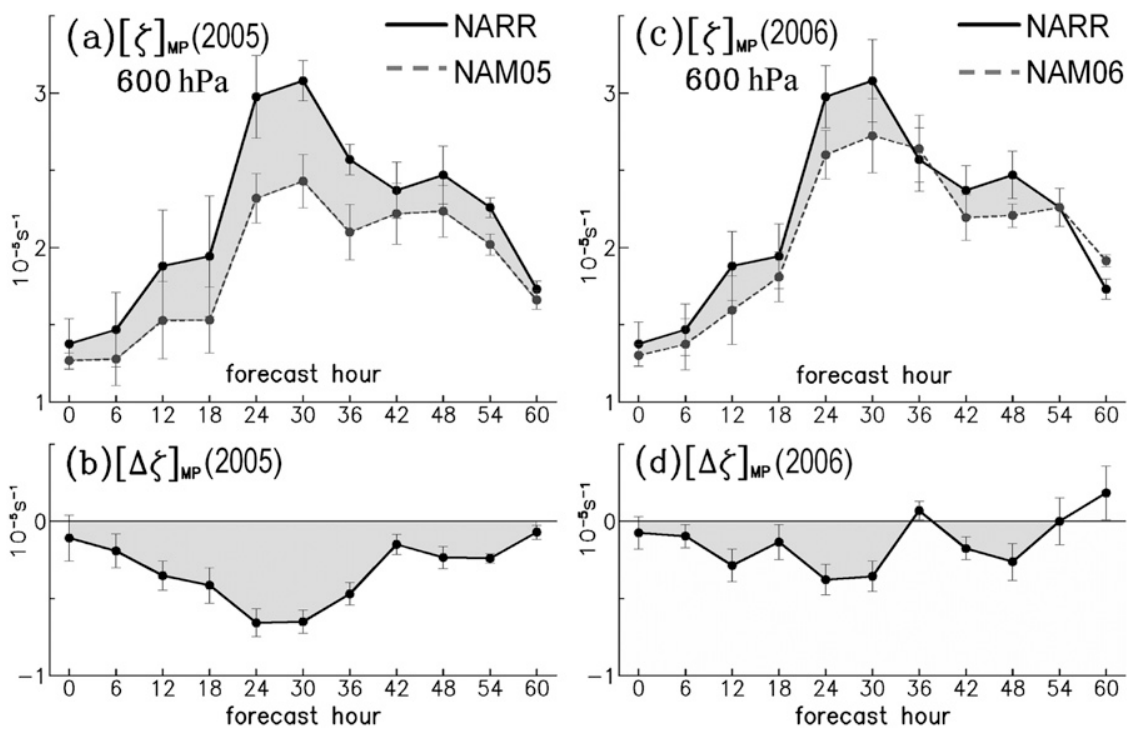

FIG. 10. (a) Time series of averaged MP vorticities at $600 \mathrm{hPa}$ throughout their 60 -h life cycle in the NARR (black solid line) and the NAM (gray dashed line) during 2005, and (b) differences in vorticity between the NARR and the NAM. (c),(d) As in (a),(b) but for 2006. Error bars are added to all time series, and the areas of difference between NARR and NAM are shaded. 

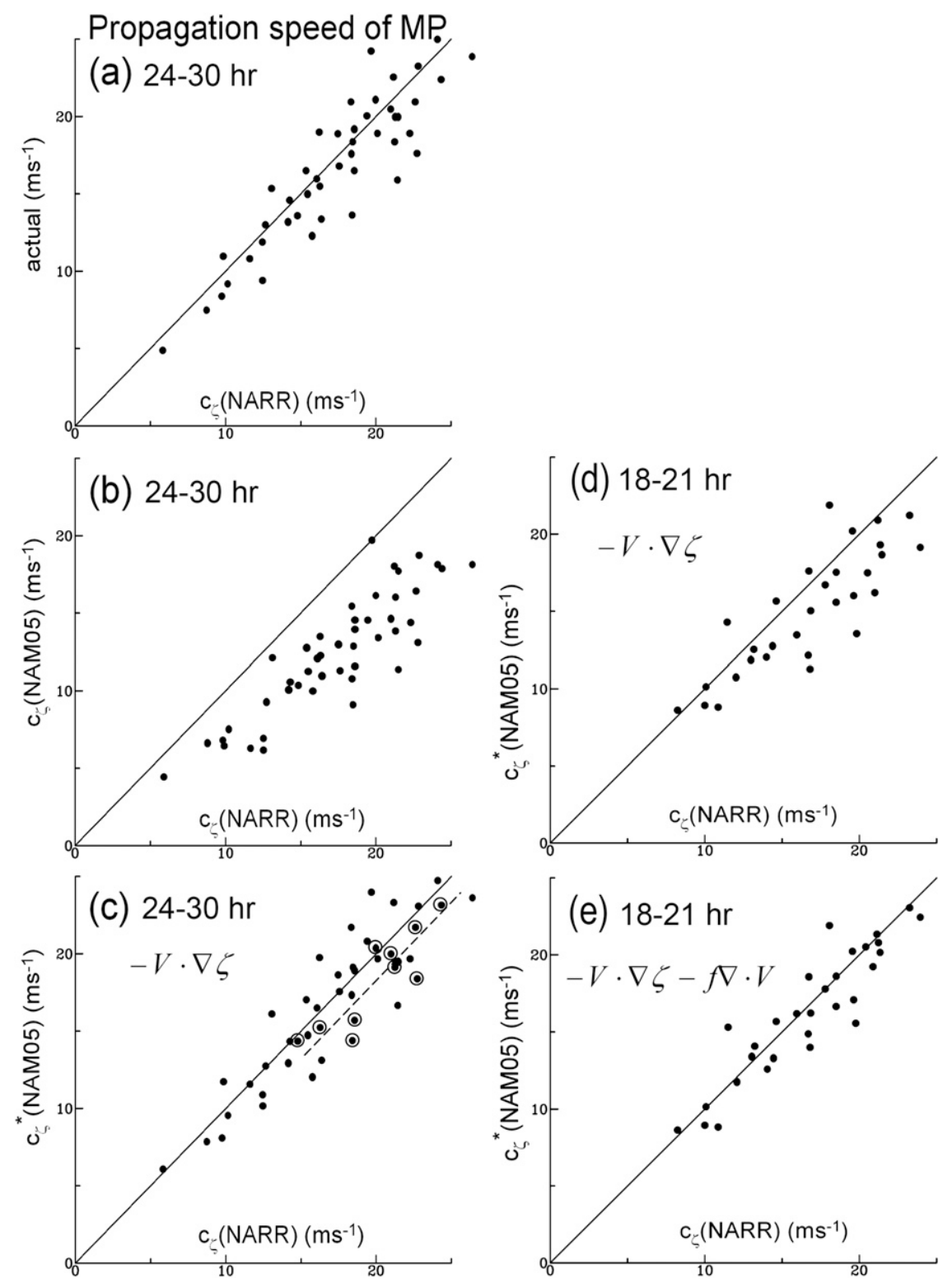

FIG. 11. Scatter diagrams of the propagation speeds of MPs during hours 24-30 with 3-h intervals in terms of (a) estimated speed of the NARR $\left[c_{\zeta}\right.$ (NARR)] vs the observed speed, (b) $c_{\zeta}$ (NARR) vs the estimated speed of NAM05 [ $c_{\zeta}$ (NAM05)], and (c) $c_{\zeta}$ (NARR) vs the reconstructed estimated speed of NAM05 [ $c_{\zeta}^{*}$ (NAM05); see text for explanations]. (d),(e) As in (c) but for MPs during hours 18 and 21 with an additional vortex stretching term in (e). The 1:1 line is added in all panels. The group of cases with the environmental wind speed $>15 \mathrm{~m} \mathrm{~s}^{-1}$ and the forecast wind speed smaller than the observed (Fig. 8) are indicated in (c) by open circles with a fitted line (dashed line).

spectrum reaches a fully developed state (Skamarock 2004) and when the model has accomplished the spinup of its microphysical variables (Clark et al. 2007). However, the highest ETS at the smallest thresholds is only 0.23 with biases under 1.5 , suggesting poor forecasts of MP-associated rainfall. Smaller ETSs with larger biases at higher thresholds further indicate that the areal rainfall coverage is overly simulated and that areas with large precipitation amounts are displaced. Next, forecasted precipitation amounts are examined through rainfall averaged within each mobile domain and filtered by six different thresholds, as shown in the scatter diagrams in Fig. 15. These amounts are 6-h accumulations, with $3 \mathrm{~h}$ before and $3 \mathrm{~h}$ after forecast hour 12 . The 


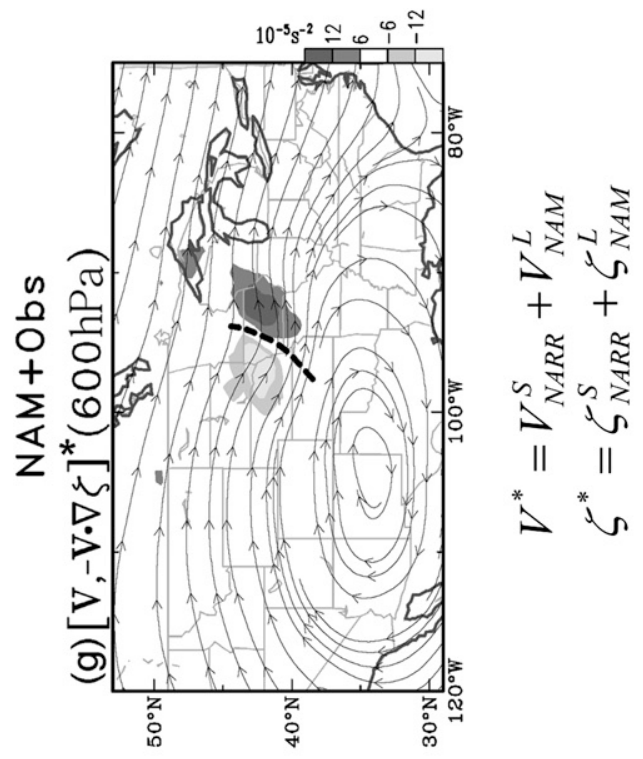

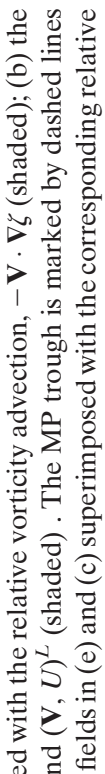
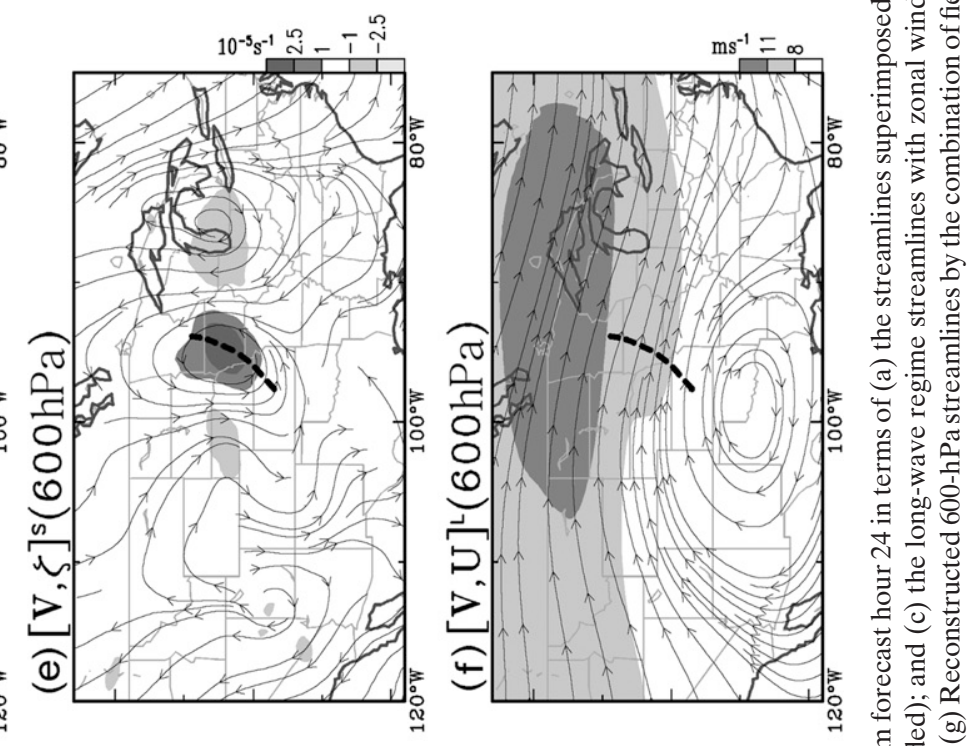

论
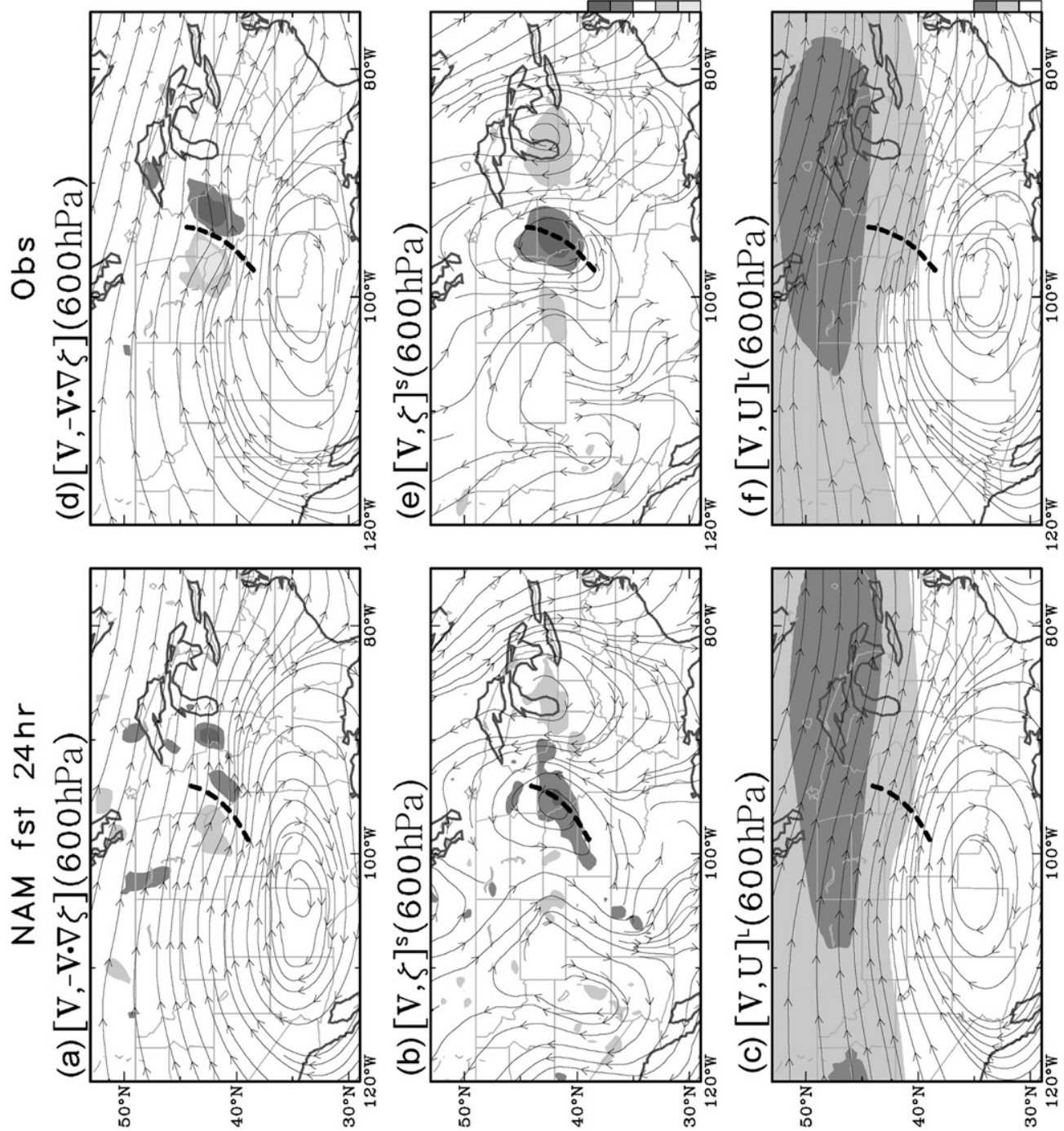

है चे

氜

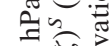
$8 \geq 0$ की $\sum$ 要 䆑 这 눙 ? 氜 $\circ$ 政 w 영 क्ञ

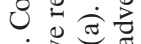
료

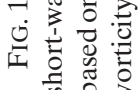



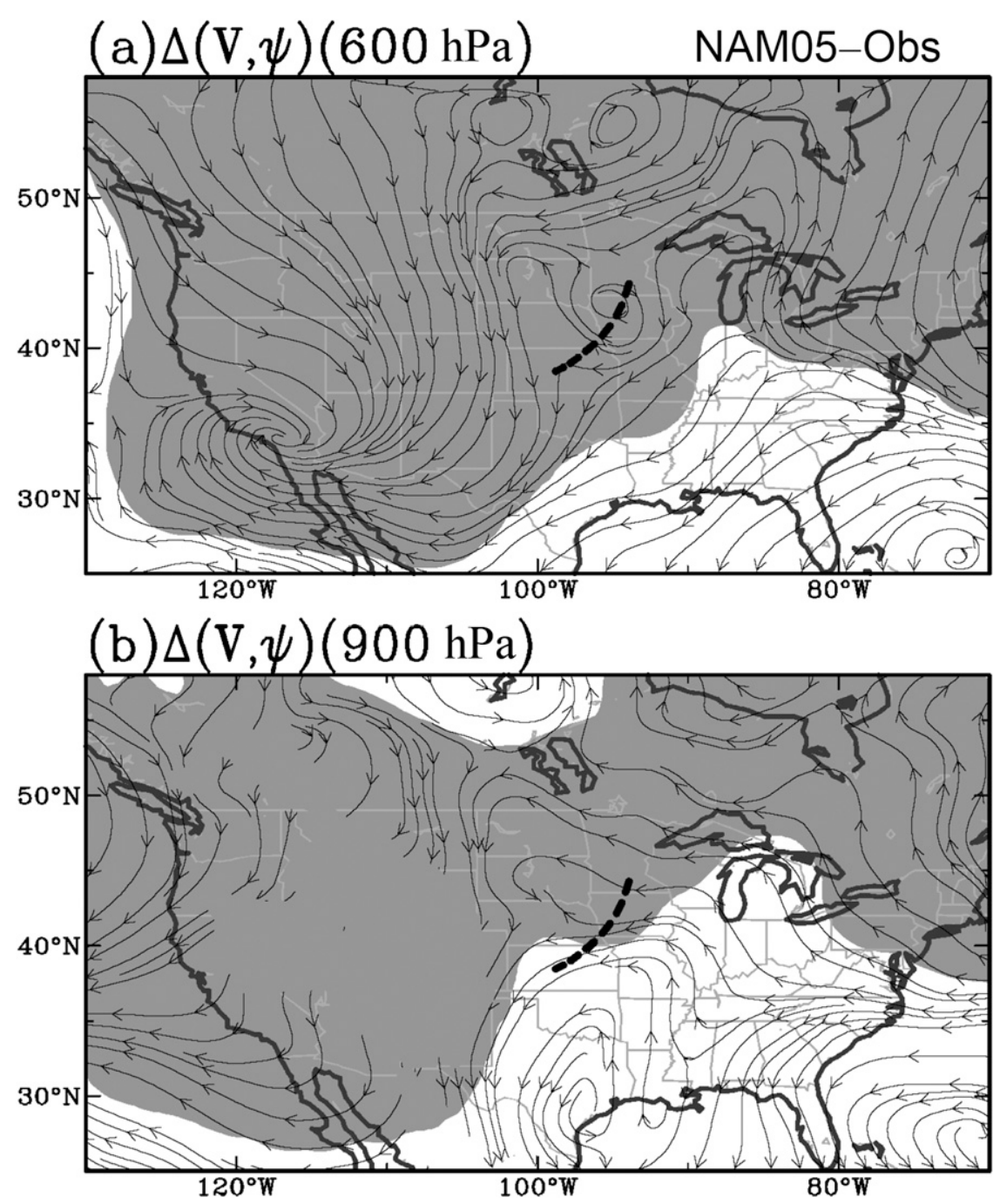

FIG. 13. Differences in composites between the NAM05 and the observations (following Figs. 12a and 12d) in terms of streamlines and streamfunction $(\Delta \psi)$ at (a) 600 and (b) $900 \mathrm{hPa}$. Areas in which $\Delta \psi \geq 0$ are shaded. Thick dashed line indicates the MP trough at $600 \mathrm{hPa}$ based on the observations.

correlation coefficients between the NAM and the observed precipitation amounts at each threshold are generally low (below 0.32), which is reflected by their scattered distributions. The shifting of precipitation toward the observations at larger thresholds indicates that both models underpredict large (or convective) rainfall.

To understand how the rainfall pattern associated with MPs is distorted in the forecasts, a synoptic examination is made. Note that, as shown in Figs. 14 and 15, the deficiencies of the rainfall forecasts of NAM05 and NAM06 differ only slightly from each other, which makes it reasonable to combine both periods for a general examination (the NAM herein). Following the composite procedure in Figs. 12a and 12d, the composite MP and its associated rainfall are shown in Fig. 16. In this case hour 12 is used and the 2006 cases are included. All cases in Table 1 are used for the MP-relative composite except for those not associated with convection. The coupling of rainfall with MPs is visible in both the observation (Fig. 16a) and the forecast (Fig. 16b), and is supported by the $95 \%$ confidence level (white contours) encircling large precipitation areas. Despite the relatively smaller amounts of the NAM precipitation, the distributions of observed and forecasted rainfall become more similar than in individual cases (e.g., Fig. 3). The similarities in the rainfall patterns suggest that the removal of the MP position error improves the precipitation forecast.

To quantitatively assess such an "improvement," the mobile ETSs of MPs are recomputed by aligning each forecasted MP with the observed one at hour 12. The 

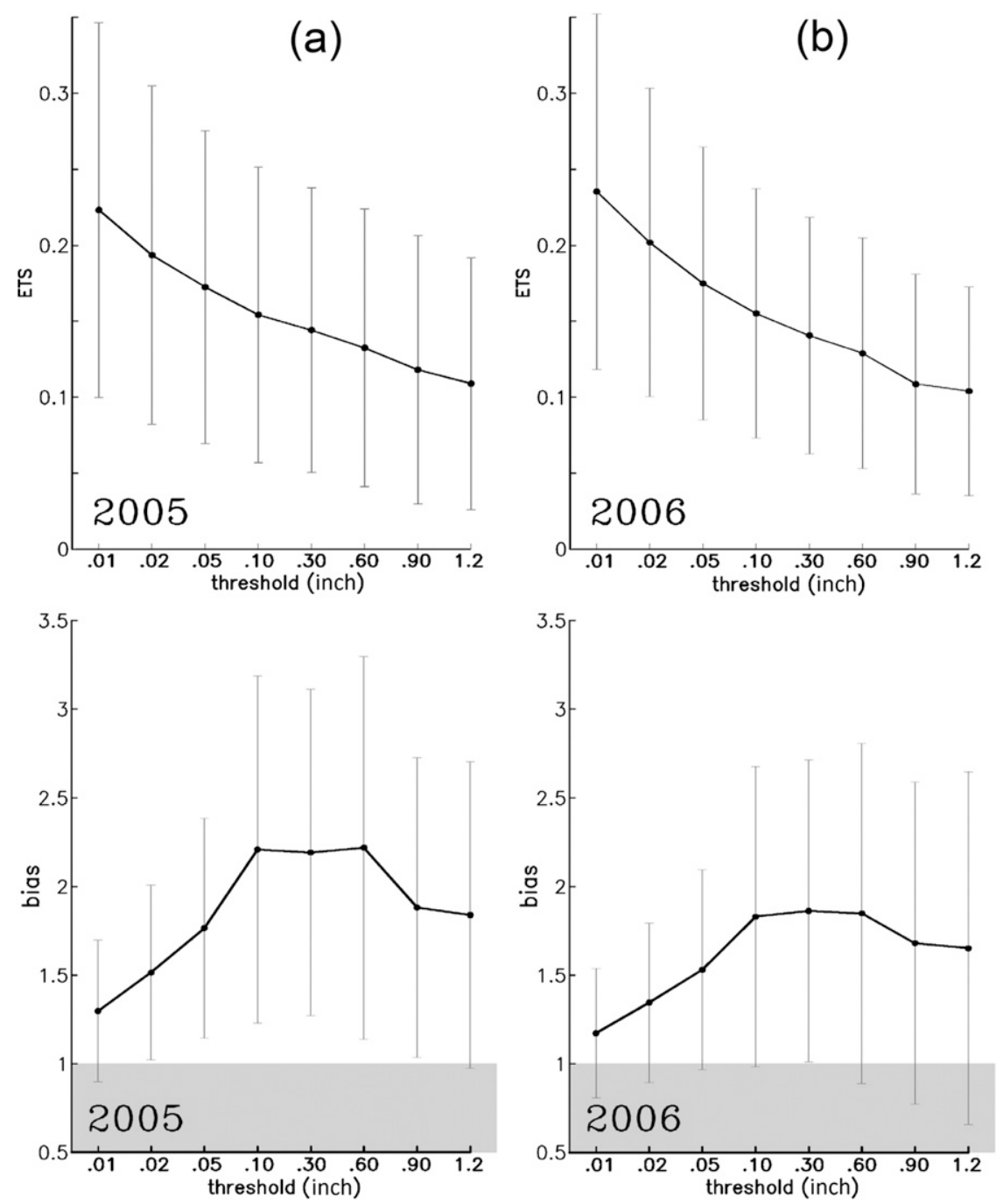

FIG. 14. (top) Mean mobile ETSs and (bottom) biases of the MP-associated precipitation forecasted by (a) NAM05 and (b) NAM06 at hour 12 with respect to different thresholds, computed over a $15^{\circ} \times 15^{\circ}$ domain centered at each observed MP during its lifetime. Error bars (thin lines) are added to the mean values (thick lines). Biases below 1 are shaded.

entire matrix of forecasted rainfall is shifted following the alignment so that the $15^{\circ} \times 15^{\circ}$ ETS domain covers the same number of grid points for both forecasted and observed rainfall. Results (Figs. 16c and 16d) show that average ETSs increase considerably while the bias scores decrease. Compared to Fig. 14, these increased scores suggest that the poor forecasts of propagating summer rainfall over the northern plains can be improved if the position-speed errors of forecasted MPs are corrected. Nevertheless, large bias scores at low thresholds still indicate a widely spread rainfall distribution, and small ETSs at large thresholds mean that the location of strong precipitation remains displaced to some extent. Comparing Figs. 16a with 16b, precipitation amounts associated with forecasted MPs also remain too small.
CPSs play a crucial role in controlling forecasted rainfall, but numerically examining different CPSs or CPS settings is beyond the scope of this study. Thus, the water vapor budget analysis, which portrays the precipitation process, is used to diagnose forecasted rainfall and provide implications for the deficiency of CPSs. The vertically integrated water vapor flux $(\mathbf{Q})$ is expressed as

$$
\mathbf{Q}=\int_{0}^{p_{s}}(\mathbf{V} q) d p
$$

where $\mathbf{V}$ is the wind field and $q$ is the specific humidity. Previous hydrological studies focusing on the central United States have recognized the importance of moisture transport by the low-level jet in precipitation (e.g., 

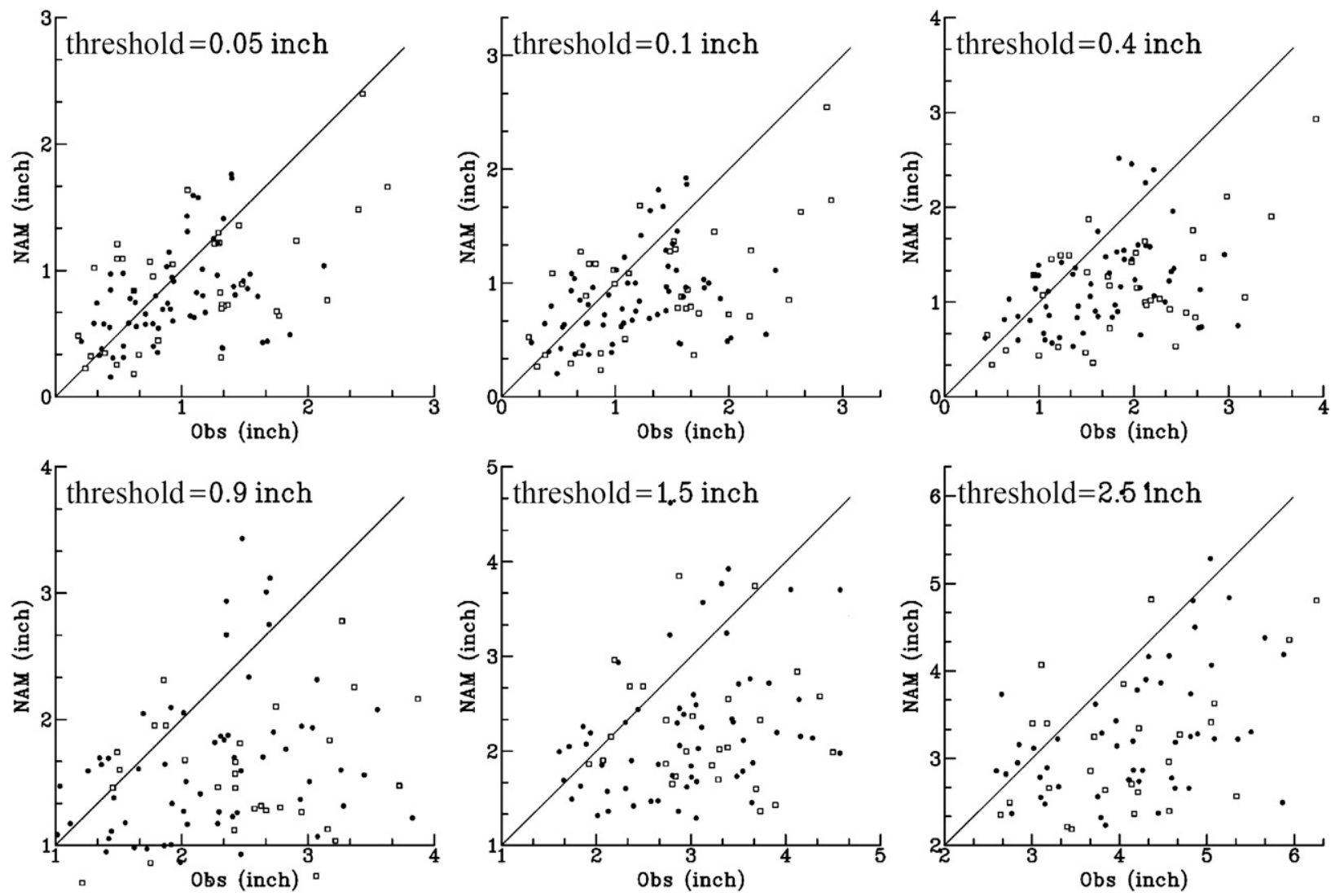

FIG. 15. Scatter diagrams showing precipitation amounts of stage IV ( $x$ axis), averaged over a $15^{\circ} \times 15^{\circ}$ domain following each observed MP, vs those of 12-h NAM forecasts ( $y$ axis) under different thresholds (which are given in the upper left of each panel). The 1:1 lines are indicated by solid lines. Values from 2005 (2006) are marked by open rectangles (solid dots).

Rasmusson 1968; Helfand and Schubert 1995), and that the precipitation associated with transient weather systems is maintained mainly through the convergence of water vapor flux (Chen and Kpaeyeh 1993), namely

$$
P \approx-\nabla \cdot \mathbf{Q} .
$$

Chen (1985) introduced the moisture flux potential $\left(\chi_{\mathbf{Q}}\right)$ by solving

$$
\chi_{\mathbf{Q}}=\nabla^{-2}\left(\nabla \cdot \mathbf{Q}_{D}\right)
$$

Equations (6) and (7) describe the maintenance mechanism of precipitation through the divergent components of the water vapor flux $\left(\mathbf{Q}_{D}\right)$ in terms of the horizontal gradients of $\chi_{\mathbf{Q}}$.

The observed $\chi_{\mathbf{Q}}$ and $\mathbf{Q}_{D}$ of the composite MP (Fig. 17a; following Fig. 16a) depict the strong convergence of the water vapor flux immediately east of the perturbation trough (dashed line) where large precipitation (Fig. 16a) is maintained. Computing $\chi_{\mathbf{Q}}$ and $\mathbf{Q}_{D}$ for the NAM (following Fig. 16b) and subtracting these variables from the observations, the differences (Fig. 17b) show that the convergence of forecasted $\chi_{\mathbf{Q}}$ is too weak ahead of the MP trough, but too strong behind it. These anomalous $\chi_{\mathbf{Q}}$ and $\mathbf{Q}_{D}$ patterns can weaken the moisture convergence ahead of the MP and thereby suppress the rainfall production. The anomalous $\chi_{\mathbf{Q}}$ convergence behind the MP may displace rainfall, which is supposed to be distributed ahead of the perturbation.

Based on Eqs. (5) and (7), winds and specific humidity are the fundamental elements involved in water vapor transport. Without having to engage in numerical experiments, the bias of the atmospheric humidity can be diagnosed through the following procedure. By replacing the NAM $q$ with the observed one in Eq. (5), a reconstructed water vapor flux $\mathbf{Q}^{*}\left[=\int_{300}^{p_{S}}\left(\mathbf{V}_{\mathrm{NAM}} q_{\mathrm{NARR}}\right) d p\right]$ and, subsequently, $\chi_{\mathbf{Q}}^{*}\left[=\nabla^{-2}\left(\boldsymbol{\nabla} \cdot \mathbf{Q}_{D}^{*}\right)\right]$ can be obtained. Figure $17 \mathrm{c}$ shows that the differences between $\chi_{\mathbf{Q}}^{*}$ and the observed $\chi_{\mathbf{Q}}$ are substantially reduced compared to Fig. 17b, meaning that $\chi_{\mathbf{Q}}^{*}$ is now closer to the observations. When $\chi_{\mathbf{Q}}^{*}$ is reconstructed by $\int_{300}^{p_{S}}\left(\mathbf{V}_{\text {NARR }} q_{\mathrm{NAM}}\right) d p$ (not shown), 

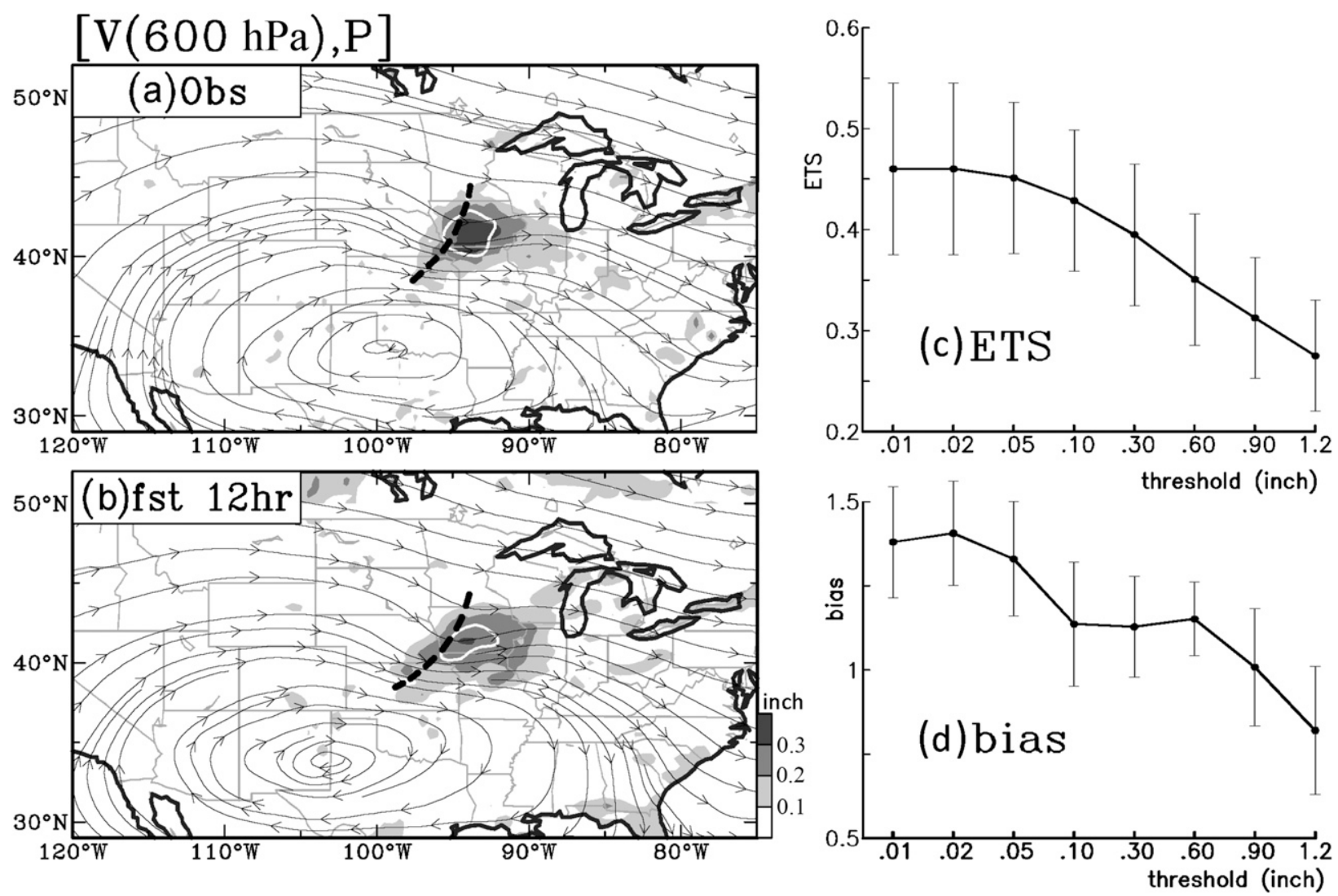

FIG. 16. Composite streamlines at $600 \mathrm{hPa}$ of the (a) NARR with stage IV rainfall and (b) 12-h NAM forecasts during July-August of 2005-06, following the procedure outlined in Figs. 12a and 12d. The 95\% confidence level of the composite precipitation is indicated by white contours surrounding large precipitation. ETSs and biases of rainfall computed in the domain outlined in (b) (dotted rectangular) are shown in (c) and (d), respectively, with different thresholds.

the anomalous pattern does not differ much from that in Fig. 17b. The similar polarity between Figs. $17 \mathrm{~b}$ and $17 \mathrm{c}$ suggests that insufficiently simulated atmospheric humidity is closely linked to the rainfall bias. Since humidity is a crucial part of the mixing parameterization, obtaining a realistic humidity profile in CPSs should be a necessary step toward improving summer precipitation forecasts in this region.

\section{c. Implications of precipitation errors on $M P$ forecasting}

Previous studies (e.g., Menard and Fritsch 1989; Hertenstein and Schubert 1991; Braun and Houze 1996) have found that latent heating within midlatitude squall lines can induce vorticity generation and form midlevel vortices, especially those associated with trailing stratiform precipitation. Intense convective storms coupled with MPs are often characterized by a squall line that accompanies trailing stratiform rainfall (e.g., Fig. 4). Since precipitation is coupled with the positive vorticity tendency of the MPs, as displayed in Figs. 12 and 16, the precipitation bias may feed back to affect the forecasts of MPs through heating.

Here, we examine such processes by use of potential vorticity (PV), as PV detects the effects of both heating and momentum transports. The isobaric PV budget equation may be written as

$$
\frac{\partial P}{\partial t}=-\nabla \cdot(P \mathbf{V})-\frac{\partial}{\partial p}(P \omega)-g \boldsymbol{\nabla} \cdot\left[\frac{1}{c_{p}} \dot{Q} \hat{\mathbf{k}}(f+\zeta)\right] .
$$

Term $1-3$, where $P$ is the Ertel $\mathrm{PV} \equiv[\mathbf{g}(f+\zeta) \cdot \nabla \theta], \theta$ is the potential temperature, $c_{p}$ is the specific heat, $\left(1 / c_{p}\right) \dot{Q}$ is the diabatic heating obtained by the residual method of the thermal dynamic equation, and the rest symbols are in their conventional formats, following Wiin-Nielsen and Sela (1971) and Raymond (1992). The frictional term is neglected. The profiles of $\left(1 / c_{p}\right) \dot{Q}$ and all terms in Eq. (8) are then averaged from a $4^{\circ} \times 4^{\circ}$ domain at the maximum leading precipitation of the MPs. We use hours 18-21 when the rapid amplification of most MPs takes 

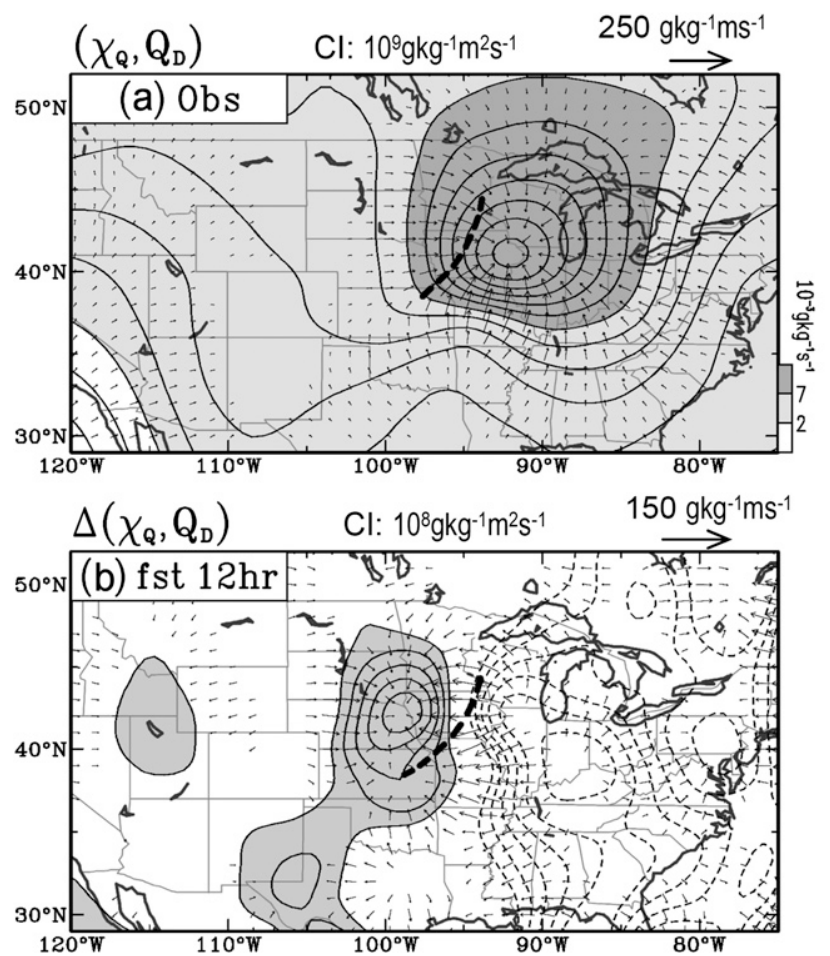

$\Delta\left(\chi_{Q}^{*}, Q_{\mathrm{D}}^{*}\right)$ using $\mathrm{q}(\mathrm{NARR})$

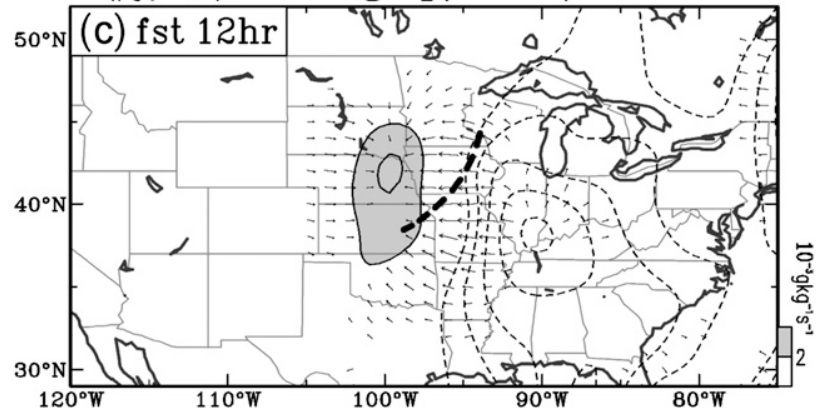

FIG. 17. (a) Composite MP in terms of the moisture flux potential $\left(\chi_{\mathbf{Q}}\right)$ and divergent components $\left(\mathbf{Q}_{D}\right.$; vectors $)$ of the water vapor flux from the NARR; (b) differences of $\chi_{\mathbf{Q}}$ and $\mathbf{Q}_{D}$ between the 12-h NAM forecasts and the NARR, $\Delta\left(\chi_{\mathbf{Q}}, \mathbf{Q}_{D}\right)$; and (c) differences between the reconstructed $\chi_{\mathbf{Q}}$ and $\mathbf{Q}_{D}$ using the observed specific humidity, $\Delta\left(\chi_{\mathbf{Q}}^{*}, Q_{D}^{*}\right)$ (see text). The trough line of MP corresponds to that in Fig. 16a. Zero contours are omitted in (b) and (c).

place, following Figs. 11d and 11e, and only include strongly convective cases in which the maximum precipitation boundary is larger than $200 \mathrm{~km}$ (see Table 1).

The diabatic heating profiles over the leading precipitation of the MPs in both the NARR and the NAM (Fig. 18a) resemble those found in the convective region of propagating squall lines where latent heating dominates at middle and upper levels (e.g., Gallus and Johnson 1991; Braun and Houze 1996). An apparent cooling bias in the NAM appears in the mid- to upper troposphere with a slight warming bias near the surface. The PV budget analysis of the NARR (Fig. 18b) shows that the maximum PV tendency occurs in the midtroposphere and is formed primarily by horizontal PV flux divergence (term 1) and secondarily by diabatic PV stretching (term 3). Vertical PV flux divergence (term 2) remains weak until $500 \mathrm{hPa}$ and then decreases with height in response to the midlevel PV maximum (Bartels et al. 1997). Here, similar to Fig. 8a, term 1 is dominated by the horizontal advection of $\mathrm{PV}(-\mathbf{V} \cdot \nabla P)$ (not shown).

In the NAM (Fig. 18c), the PV budget structure is generally consistent with that of the NARR, but all terms are noticeably smaller. The weak diabatic PV stretching and weak vertical PV flux divergence are consistent with the weak precipitation (cf. Fig. 16b). These biases altogether result in a $28 \%$ "deficit" of the PV tendency (relative to the NARR) within the 550650-hPa layer, where the maximum $\partial P / \partial t$ occurs. The bias in the PV tendency resulting from diabatic PV stretching (term 3; negative bias) is largely canceled by that from the vertical PV transport (term 2; positive bias), particularly from $600 \mathrm{hPa}$ above. Thus, the net $\partial P / \partial t$ bias is primarily contributed from the bias in horizontal PV advection. This observation agrees with the vorticity budget analysis (Fig. 8b) in which horizontal vorticity advection plays a major role in the $\zeta_{t}$ bias.

To examine the impacts of the heating bias on $\partial P / \partial t$, the PV and the thermal dynamic equation in the NAM are recalculated by replacing $\theta$ with the NARR, yielding $P^{*}=\left[\mathbf{g}\left(f+\zeta_{\mathrm{NAM}}\right) \cdot \nabla \theta_{\mathrm{NARR}}\right]$ and $\left(1 / c_{p}\right) \dot{Q}^{*}$ (the residual), respectively. A "reconstructed" $\mathrm{PV}$ tendency, $\partial P^{*} / \partial t$, is then obtained from the three terms in Eq. (8) using $P^{*}$ and $\left(1 / c_{p}\right) \dot{Q}^{*}$. Note that this approach does not exclude the bias in vertical velocity $(\omega)$, which links to convection, but it does help to sharpen the focus on the bias in heating. Compared with $\partial P / \partial t$ in the NAM, $\partial P^{*} / \partial t$ (Fig. 18d) is closer to the NARR while the bias at $550-650 \mathrm{hPa}$ is now reduced to $22 \%$. This gives a crude 

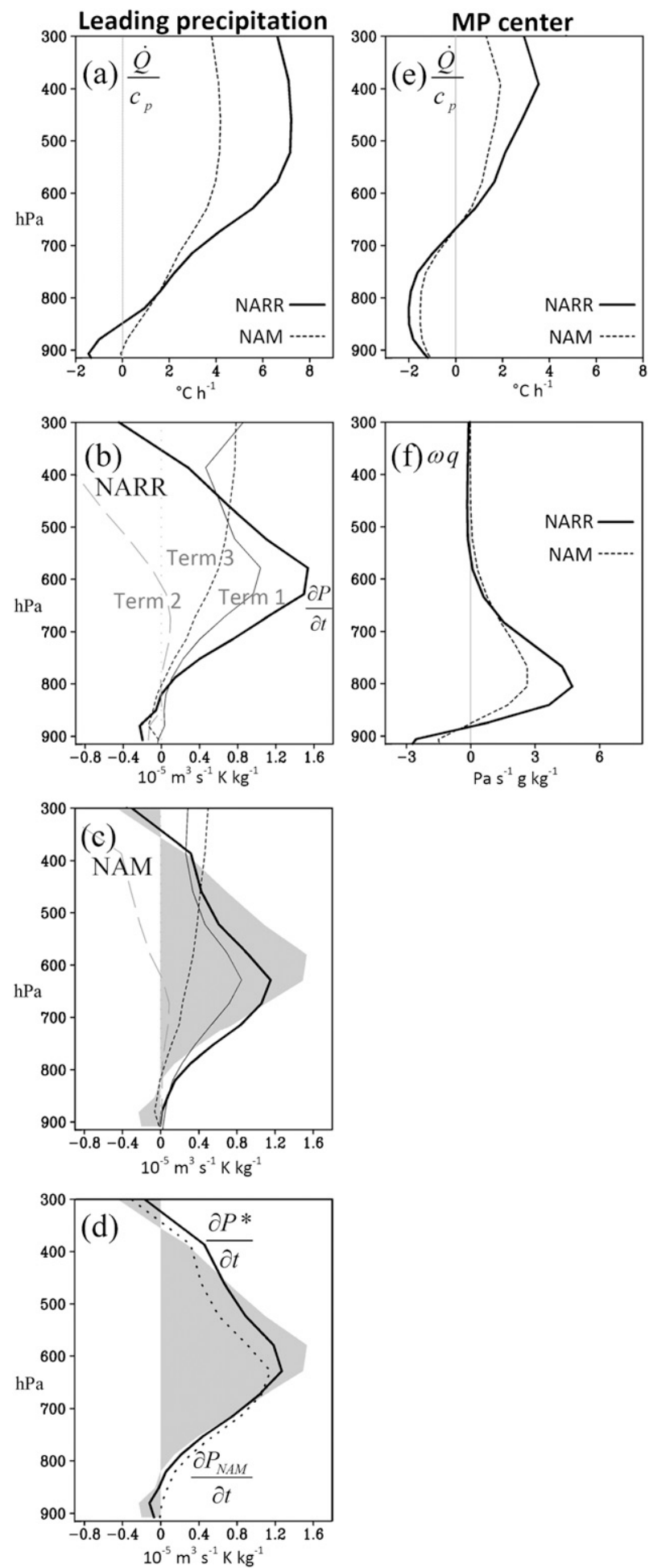

FIG. 18. Composite vertical profiles of (a) diabatic heating of the NARR (solid line) and the NAM (dashed line), (b) the PV budget of the NARR with each term indicated, (c) the PV budget of the NAM following (b), and (d) the PV tendency of the NAM (dotted line) and $\partial P^{*} / \partial t$ (solid line). All terms are averaged within a $4^{\circ} \times 4^{\circ}$ domain over the leading precipitation area as in Fig. 16. The PV tendency of the NARR is shaded in (c) and (d) for comparison. indication that $21 \%[=(28-22) / 28 \%]$ of the bias in the midlevel PV tendency may result from the bias in heating (or temperature profile, to be specific) relative to the leading precipitation of MPs, and that the rest $(79 \%)$ of the bias is presumably related to kinematic fields. Such a heating bias may help explain why the perturbation vorticity errors (Fig. 10b) grow most quickly between hours 18 and 24, as well as the increased bias in vortex stretching during this time period (Fig. 11e). Because the PV tendency dictates the movement of a symmetric PV column [such as idealized tropical cyclones; Chan (1984)], weak heating that reduces the PV tendency is expected to slow the MPs. Numerical investigation will be necessary to quantitatively assess the impacts of the precipitation bias on the MP movement.

Over the MP center, the stratified $\left(1 / c_{p}\right) \dot{Q}$ profile (Fig. 18e) is similar to that found in the stratiform region of propagating squall lines (cf. Fig. 9 in Braun and Houze 1996), with cooling and downward vertical moisture flux ( $\omega \mathrm{q}$; Fig. 18f) at lower levels. These profiles depict moisture downdrafts that are typically maintained by evaporational cooling and precipitation drag. Moist downdrafts can accelerate the deep convection (Zhang and Fritsch 1988; Zhang 1992); however, the weak low-level cooling and $\omega \mathrm{q}$ in the NAM indicate weakly forecasted moisture downdrafts. In addition, the rear downward branch of the MPs sometimes occurs in conjunction with low-level downdrafts in the trailing stratiform area (WCC). According to the convergent $\chi_{\mathbf{Q}}$ anomalies behind the MP (Fig. 17b), this rear downward branch is also too weak in the NAM. The bulk of such biases may additionally slow MCSs coupled with MPs, because the lack of moist downdrafts in the forecast models tends to decelerate the convection (Bélair and Zhang 1997).

\section{d. Implications from the summer climatology}

The systematic biases of a numerical forecast model usually reflect the deficiencies of the model physics or dynamics. A convenient way of identifying such deficiencies is through the examination of the model climatology. Here, the NAM climatology of the circulation and rainfall is constructed using hour 24 initialized at every 6-h cycle (0000, 0600, 1200, and 1800 UTC) throughout July and August of 2005-06. Possible interannual variation is not the focus of this study, so the years of 2005 and 2006 are combined for the examination. The biases of the circulation and rainfall are illustrated in

(e),(f) The composite diabatic heating and vertical water vapor flux profiles, respectively, in the NARR (solid line) and the NAM (dashed line) over the MP center. 


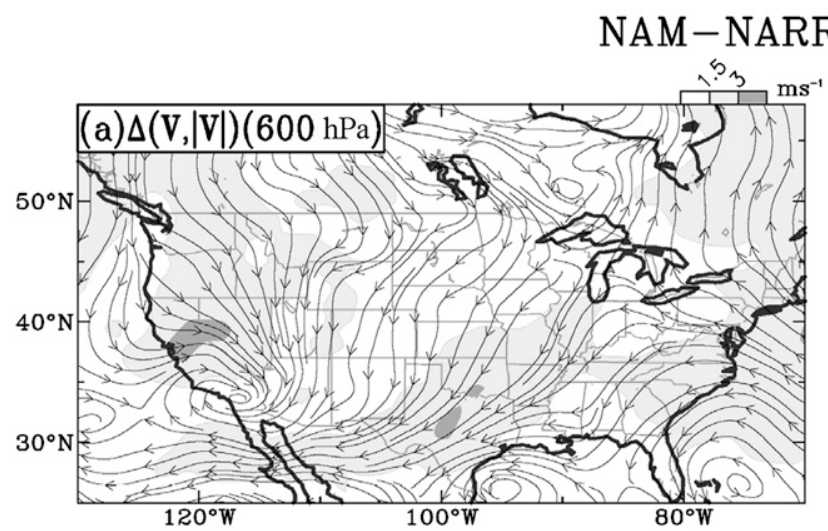

\section{$\mathrm{JA}(05-06)$}
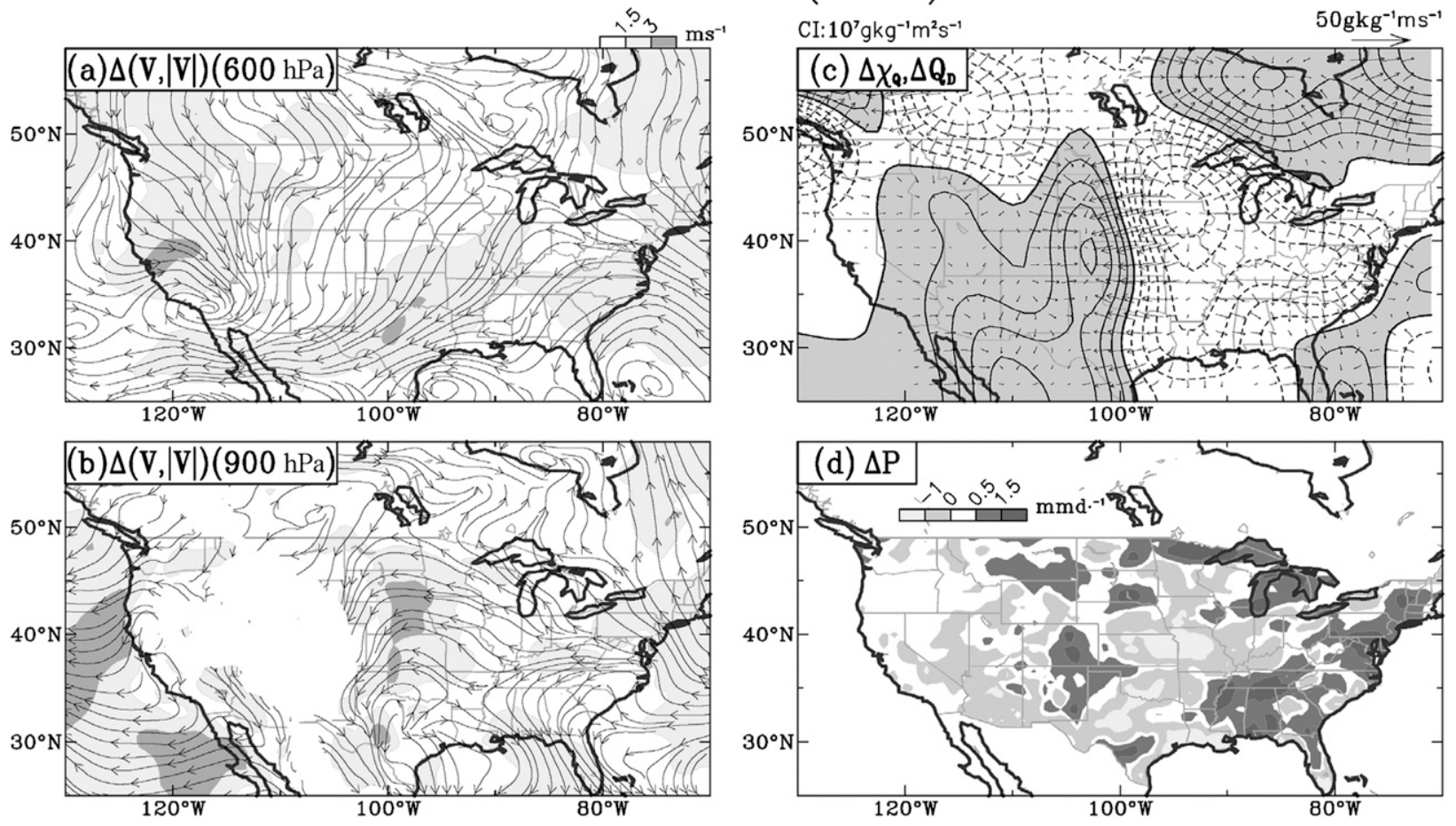

FIG. 19. Differences in the mean (a) 600- and (b) 900-hPa streamlines superimposed with wind speed, (c) $\chi_{\mathbf{Q}}$ (moisture flux potential) and $\mathbf{Q}_{D}$, and (d) precipitation between the 24-h NAM forecasts and the NARR during July-August of 2005-06.

Fig. 19 by the differences in the forecasted climatological fields from the observed ones.

In the midtroposphere (600 hPa; Fig. 19a), the anticyclonic circulation covering the western United States indicates that the intensity of the North American anticyclone (Fig. 1) is too strong. The northeasterly flow over the central United States coincides with the undersimulated wind speed depicted in Fig. 9. At low levels (900 hPa; Fig. 19b), the cyclonic circulation over the central southern United States implies that the westward extension of the Bermuda anticyclone, along with the Great Plains low-level jet, are too weak in the NAM. These circulation anomalies greatly resemble those in the MP composites (Fig. 13), suggesting that the biases of the large-scale circulation (i.e., strong anticyclone and easterly anomalies) contribute to the weak vorticity and ambient flows of the MPs, as revealed in section $4 a$.

Previous studies focusing on the interannual variations of the hydroclimate pointed out that the rainfall reduction in the central United States is linked to anomalous northerly flow in the middle to upper troposphere and weakened low-level jets, which lead to weak convergence of the water vapor flux in the central plains (e.g., Wallace and Gutzler 1981; Mo et al. 1997). This process coincides with the NAM's tendency to produce insufficient MP rainfall, because the anomalous anticyclone, the weakened low-level jet (Fig. 19b), and the divergent $\chi_{\mathbf{Q}}$ anomalies (Fig. 19c) together resemble the dry-season circulation anomalies. The precipitation deficit over the central plains (Fig. 19d) is in good agreement with the suppressed moisture convergence there (Fig. 19c). The climatological analysis indicates that the systematic biases associated with MPs involve all scales and are rather complicated. On the other hand, it also suggests that correcting the biases in the model climatology should help remove the systematic biases in MP forecasting. MCS forecasting remains a challenge, however, because the root cause of summer precipitation errors is widely believed to be the shortcomings associated with CPSs (Davis et al. 2003; Fritsch and Carbone 2004), which are still a necessity in the operational NAM and are difficult to improve.

\section{Concluding remarks}

The upper-level circulation during July and August is characterized by the North American anticyclone with northwesterly flow across the northern plains. Under such a circulation pattern, which is considered weakly forced, midtropospheric perturbations with a subsynoptic scale often are initiated over the Rockies and propagate across the northern plains. These MPs are capable of 
inducing long-lived, progressive MCSs. In view of the unsatisfactory performance of the forecast models in simulating summer propagating rainfall, this study examines the operational forecasts of MPs by the NAM during July and August of 2005-06. Comparisons are carried out on the NAM output with the NARR and the stage IV rainfall data.

The NAM performs reasonably well in capturing the genesis and development of MPs, as well as the general thermodynamic structure of the associated MCSs. However, the NAM forecast of MPs exhibits two types of systematic errors: 1) the propagation speed of MPs is too low and 2) the precipitation associated with MPs is displaced and too small.

\section{a. MP propagation}

The NAM is capable of simulating the cyclonic circulation and eastward propagation of MPs. The genesis locations and occurrence frequencies of MPs in 2005 and 2006 were handled relatively well by the NAM. On the other hand, forecasted MPs tend to propagate more slowly than what is actually observed. Such a speed bias coincides with the simulated perturbation vorticity that is constantly too weak. In addition, the forecasted ambient flow speed tends to be too low when the flow is strong. Weak relative vorticity combined with low flow speed lead to weak horizontal vorticity advection, which aids in slowing the perturbations. When convective storms associated with MPs are developing, biases in vortex stretching and diabatic heating can reduce the perturbation growth and additionally slow MPs in the forecast. Furthermore, the midsummer anticyclone simulated by the NAM is too strong. This strong pseudoanticyclone forms an unfavorable background circulation, which may suppress the cyclonic vorticity developing inside. Such biases are particularly pronounced in 2005 when the Eta Model represented the NAM.

\section{b. Precipitation forecast}

A mobile approach is adopted to compute the skill scores for the MP-associated precipitation. The skill scores are generally low due to the position errors of the forecasted MPs displacing rainfall. After realigning the forecasted MPs with observed ones, the ETSs of the MP-associated rainfall significantly improved. In other words, the NAM simulates the MP-MCS coupling relatively well, but the speed bias of the MPs hampers the precipitation forecasts. Another source of bias causing the low skill scores is the underpredicted precipitation amounts. We find that the rainfall production process in the NAM, which is governed by CPSs, reveals insufficient atmospheric moisture accompanied by weak convergence of the water vapor flux.
The bias for this weak precipitation is particularly a concern when forecasting MPs accompanying strong or derecho-producing MCSs. This is because the growth and propagation of such MPs are further complicated by convective heating through midlevel PV generation. This situation poses a great challenge to the operational NAM model, which still suffers from the difficulties of CPSs in predicting convection at the correct location with correct heating profiles.

The listed biases should be further studied through sensitivity tests of CPSs and model grid spacings, since these elements remain crucial in humidity and precipitation simulations. Clark et al. (2007) found that the 5-km grid spacing in the WRF model, which omits the use of CPSs and explicitly resolves convection processes, considerably improves the propagating signature of the midwestern rainfall compared to coarser grid spacings, which must engage CPSs. Their results imply that excluding CPSs may be a practical way of improving the forecasting of MPs.

Acknowledgments. This study was conducted under the support of the ISU Baker Endowment Fund 497-4139-15-3803 publication funding was provided by Iowa State University Extension Climate Research and Information Project. We are grateful to Adam Clark for his valuable comments, as well as to Marty Booth for editorial assistance. Comments offered by Dr. Da-Lin Zhang and two reviewers have been helpful and are appreciated.

\section{REFERENCES}

Barnes, S. L., F. Caracena, and A. Marroquin, 1996: Extracting synoptic-scale diagnostic information from mesoscale models: The Eta Model, gravity waves, and quasigeostrophic diagnostics. Bull. Amer. Meteor. Soc., 77, 519-528.

Bartels, D. L., J. M. Brown, and E. I. Tollerud, 1997: Structure of a midtropospheric vortex induced by a mesoscale convective system. Mon. Wea. Rev., 125, 193-211.

Bélair, S., and D. L. Zhang, 1997: A numerical study of the alongline variability of a frontal squall line during PRE-STORM. Mon. Wea. Rev., 125, 2544-2561.

Bentley, M. L., and T. L. Mote, 1998: A climatology of derechoproducing mesoscale convective systems in the central and eastern United States, 1986-95. Part I: Temporal and spatial distribution. Bull. Amer. Meteor. Soc., 79, 2527-2540.

Bernardet, L. R., L. B. Nance, H.-Y. Chuang, A. Loughe, M. Demirtas, S. Koch, and R. Gall, 2005: The Developmental Testbed Center Winter Forecasting Experiment (DWFE). Preprints, 21st Conf. on Weather Analysis and Forecasting/ 17th Conf. on Numerical Weather Prediction, Washington, DC, Amer. Meteor. Soc., 7.1. [Available online at http://ams.confex. com/ams/pdfpapers/94730.pdf.]

Black, T. L., 1994: The new NMC mesoscale Eta Model: Description and forecast examples. Wea. Forecasting, 9, 265-278. 
Bosart, L. F., and F. Sanders, 1981: The Johnstown flood of July 1977: A long-lived convective system. J. Atmos. Sci., 38, 16161642.

Braun, S. A., and R. A. Houze, 1996: The heat budget of a midlatitude squall line and implications for potential vorticity production. J. Atmos. Sci., 53, 1217-1240.

Carbone, R. E., J. D. Tuttle, D. A. Ahijevych, and S. B. Trier, 2002: Inferences of predictability associated with warm season precipitation episodes. J. Atmos. Sci., 59, 2033-2056.

Carr, L. E., and R. L. Elsberry, 1995: Monsoonal interactions leading to sudden tropical cyclone track changes. Mon. Wea. Rev., 123, 265-290.

Céron, J. P., and J. F. Guérémy, 1999: Validation of the space-time variability of African easterly waves simulated by the CNRM GCM. J. Climate, 12, 2831-2855.

Chan, J. C.-L., 1984: An observational study of the physical processes responsible for tropical cyclone motion. J. Atmos. Sci., 41, 1036-1048

Changnon, S. A., and K. E. Kunkel, 1999: The record 1996 rainstorm at Chicago. J. Appl. Meteor., 38, 257-265.

Chen, T.-C., 1985: Global water vapor flux and maintenance during FGGE. Mon. Wea. Rev., 113, 1801-1819.

— associated with the low-level jet of the Great Plains. Mon. Wea. Rev., 121, 416-420.

Clark, A. J., W. A. Gallus Jr., and T.-C. Chen, 2007: Comparison of the diurnal precipitation cycle in convective-resolving and non-convection-resolving mesoscale models. Mon. Wea. Rev., 135, 3456-3473.

Coniglio, M. C., D. J. Stensrud, and M. B. Richman, 2004: An observational study of derecho-producing convective systems. Wea. Forecasting, 19, 320-337.

Davis, C. A., and S. B. Trier, 2007: Mesoscale convective vortices observed during BAMEX. Part I: Kinematic and thermodynamic structure. Mon. Wea. Rev., 135, 2029-2049.

— D. A. Ahijevych, and S. B. Trier, 2002: Detection and prediction of warm season midtropospheric vortices by the Rapid Update Cycle. Mon. Wea. Rev., 130, 24-42.

- , K. W. Manning, R. E. Carbone, S. B. Trier, and J. D. Tuttle, 2003: Coherence of warm-season continental rainfall in numerical weather prediction models. Mon. Wea. Rev., 131, 2667-2679.

Du, J., S. L. Mullen, and F. Sanders, 1997: Short-range ensemble forecasting of quantitative precipitation. Mon. Wea. Rev., 125, 2427-2459.

Fritsch, J. M., and R. E. Carbone, 2004: Improving quantitative precipitation forecasts in the warm season: A USWRP research and development strategy. Bull. Amer. Meteor. Soc., 85, 955-965.

Helfand, H. M., and S. D. Schubert, 1995: Climatology of the simulated Great Plains low-level jet and its contribution to the continental moisture budget of the United States. J. Climate, 8, 784-806.

Hertenstein, R. F., and W. H. Schubert, 1991: Potential vorticity anomalies associated with squall lines. Mon. Wea. Rev., 119, $1663-1672$.

Hilgendorf, E. R., and R. H. Johnson, 1998: A study of the evolution of mesoscale convective systems using WSR-88D data. Wea. Forecasting, 13, 437-452.

Holton, J. R., 2004: An Introduction to Dynamic Meteorology. 4th ed. Academic Press, $535 \mathrm{pp}$.

Galarneau, T. J., L. F. Bosart, C. A. Davis, and R. McTaggartCowan, 2009: Baroclinic transition of a long-lived mesoscale convective vortex. Mon. Wea. Rev., 137, 562-584.
Gallus, W. A., and R. H. Johnson, 1991: Heat and moisture budgets of an intense midlatitude squall line. J. Atmos. Sci., 48, 122-146.

Grumm, R. H., and A. L. Siebers, 1990: Systematic model forecast errors of surface cyclones in the NGM and AVN, January 1990. Wea. Forecasting, 5, 672-682.

Jankov, I., and W. A. Gallus, 2004: MCS rainfall forecast accuracy as a function of large-scale forcing. Wea. Forecasting, 19, 428-439.

Johns, R. H., 1982: A synoptic climatology of northwest flow severe weather outbreaks. Part I: Nature and significance. Mon. Wea. Rev., 110, 1653-1663.

_ 1984: A synoptic climatology of northwest flow severe weather outbreaks. Part II: Meteorological parameters and synoptic patterns. Mon. Wea. Rev., 112, 449-464.

_- 1993: Meteorological conditions associated with bow echo development in convective storms. Wea. Forecasting, 8, 294-299.

— induced windstorms. Wea. Forecasting, 2, 32-49.

Lin, Y., and K. E. Mitchell, 2005: The NCEP Stage II/IV hourly precipitation analyses: Development and applications. Preprints, 19th Conf. on Hydrology, San Diego, CA, Amer. Meteor. Soc., 1.2. [Available online at http://ams.confex.com/ams/ pdfpapers/84484.pdf.]

Liu, C., M. W. Moncrieff, J. D. Tuttle, and R. E. Carbone, 2006: Explicit and parameterized episodes of warm-season precipitation over the continental United States. Adv. Atmos. Sci., 23, 91-105.

Maddox, R. A., 1983: Large-scale meteorological conditions associated with midlatitude, mesoscale convective complexes. Mon. Wea. Rev., 111, 1475-1493.

_ C. F. Chappell, and L. R. Hoxit, 1979: Synoptic and mesoalpha scale aspects of flash flood events. Bull. Amer. Meteor. Soc., 60, 115-123.

Marchok, T., R. Rogers, and R. Tuleya, 2007: Validation schemes for tropical cyclone quantitative precipitation forecasts: Evaluation of operational models for United States landfalling cases. Wea. Forecasting, 22, 726-746.

McBride, J. L., and E. E. Ebert, 2000: Verification of quantitative precipitation forecasts from operational numerical weather prediction models over Australia. Wea. Forecasting, 15,103-121.

Menard, R. D., and J. Fritsch, 1989: A mesoscale convective complex-generated inertially stable warm core vortex. Mon. Wea. Rev., 117, 1237-1261.

Mesinger, F., and Coauthors, 2006: North American Regional Reanalysis. Bull. Amer. Meteor. Soc., 87, 343-360.

Mo, K.-C., J. N. Paegle, and R. W. Higgins, 1997: Atmospheric processes associated with summer floods and droughts in the central United States. J. Climate, 10, 3028-3046.

Olson, D. A., N. W. Junker, and B. Korty, 1995: Evaluation of 33 years of quantitative precipitation forecasting at the NMC. Wea. Forecasting, 10, 498-511.

Olsson, P. Q., and W. R. Cotton, 1997: Balanced and unbalanced circulations in a primitive equation simulation of a midlatitude MCC. Part I: The numerical simulation. J. Atmos. Sci., 54, 457-478.

Peng, M. S., and S. W. Chang, 1996: Impacts of SSM/I-retrieved rainfall rates on numerical prediction of a tropical cyclone. Mon. Wea. Rev., 124, 1181-1198.

Rasmusson, E. M., 1968: Atmospheric water vapor transport and the water balance of North America. Mon. Wea. Rev., 96, 720-734.

Raymond, D. J., 1992: Nonlinear balance and potential-vorticity thinking at large Rossby number. Quart. J. Roy. Meteor. Soc., 118, 987-1015. 
Schaefer, J. T., 1990: The critical success index as an indicator of warning skill. Wea. Forecasting, 5, 570-575.

Schumacher, R. S., and R. H. Johnson, 2008: Mesoscale processes contributing to extreme rainfall in a midlatitude warm-season flash flood. Mon. Wea. Rev., 136, 3964-3986.

Skamarock, W. C., 2004: Evaluating mesoscale NWP models using kinetic energy spectra. Mon. Wea. Rev., 132, 3019-3032.

Stensrud, D. J., 2007: Parameterization Schemes-Keys to Understanding Numerical Weather Prediction Models. Cambridge Press, $459 \mathrm{pp}$

, and J. M. Fritsch, 1994: Mesoscale convective systems in weakly forced large-scale environments. Part II: Generation of a mesoscale initial condition. Mon. Wea. Rev., 122, 2068-2083.

Trier, S. B., C. A. Davis, D. A. Ahijevych, M. L. Weisman, and G. H. Bryan, 2006: Mechanisms supporting long-lived episodes of propagating nocturnal convection within a 7-Day WRF model simulation. J. Atmos. Sci., 63, 2437-2461.

Tuttle, J. D., and C. A. Davis, 2006: Corridors of warm season precipitation in the central United States. Mon. Wea. Rev., 134, 2297-2317.
Uccellini, L. W., and D. R. Johnson, 1979: The coupling of upper and lower tropospheric jet streaks and implications for the development of severe convective storms. Mon. Wea. Rev., 107, 682-703.

Wallace, J. M., and D. S. Gutzler, 1981: Teleconnections in the geopotential height field during the Northern Hemisphere winter. Mon. Wea. Rev., 109, 784-812.

Wang, S.-Y., T.-C. Chen, and J. Correia, 2009: Climatology of summer midtropospheric perturbations in the U.S. Northern Plains. Part I: Influence on northwest flow severe weather outbreaks. Climate Dyn., in press.

Wiin-Nielsen, A., and J. Sela, 1971: On the transport of quasigeostrophic potential vorticity. Mon. Wea. Rev., 99, 447-459.

Zhang, D. L., 1992: The formation of a cooling-induced mesovortex in the trailing stratiform region of a midlatitude squall line. Mon. Wea. Rev., 120, 2763-2785.

_, and J. M. Fritsch, 1988: Numerical simulation of the meso- $\beta$ scale structure and evolution of the 1977 Johnstown flood. Part III: Internal gravity waves and the squall line. J. Atmos. Sci., 45, 1252-1268. 